\title{
QUASIGEODESIC ANOSOV FLOWS AND HOMOTOPIC PROPERTIES OF FLOW LINES
}

\author{
SÉRGIO R. FENLEY
}

\begin{abstract}
A nonsingular flow is quasigeodesic when all flow lines are efficient in measuring distances in relative homotopy classes. We analyze quasigeodesic Anosov flows in 3-manifolds which have negatively curved fundamental group. We prove that the lifts of the stable and unstable foliations to the universal cover are foliations with branching, that is, they have non-Hausdorff leaf space. Furthermore any branching is associated to freely homotopic closed orbits of the flow in the manifold and there are finitely many such branching leaves up to covering translations. Using this we prove that the limit sets of the stable and unstable leaves in the universal cover cannot be Jordan curves nor the whole sphere. Identifications of ideal points of leaves are also described using freely homotopic orbits. Finally, for any Anosov flow in such manifolds, we prove the existence of uncountably many (infinitely many of which are closed) $K$ quasigeodesic orbits for $K$ big enough. The key tool is the analysis of freely homotopic closed orbits, which are completely characterized for general Anosov flows.
\end{abstract}

\section{Introduction}

The primary goal of this article is to study metric properties of flow lines of Anosov flows in closed 3-manifolds. The two classical families in dimension 3, namely suspensions of Anosov diffeomorphisms of the two-dimensional torus (briefly suspensions) and geodesic flows on the unit tangent bundle of surfaces of negative curvature (geodesic flows), have the following property: in the appropriate metrics, the flow lines are geodesic. Since the manifolds are compact, the flow lines cannot be minimal geodesics in the usual sense. But they are minimal in relative homotopy classes, which is the same as being minimal geodesics when lifted to the universal cover.

A natural question is to decide which Anosov flows have this metric property. The requirement that flow lines be minimal geodesics is too strong and depends on the metric. Therefore relax this to the quasi-

Received December 7, 1992 and, in revised form, March 2, 1994. Research partially supported by NSF grants DMS-9201744 and DMS-9306059. 
geodesic property: a quasigeodesic curve is one that is efficient, up to a bounded multiplicative distortion, in measuring distances in relative homotopy classes. This is independent of the metric in the manifold. A flow is quasigeodesic if all flow lines are quasigeodesics.

In hyperbolic manifolds quasigeodesics are a bounded distance from true geodesics (in the universal cover) and so are strongly related to the geometry in the large of the manifold. This makes them extremely important as was demonstrated by Thurston [35], [25] in the proof of the hyperbolization theorem. The classical families of Anosov flows are in toroidal 3-manifolds, which are not hyperbolic, but a large class of Anosov flows has been constructed in hyperbolic 3-manifolds by Goodman [20] and Christy [9], using Dehn surgery on closed orbits of suspensions [16]. As opposed to the classical families, very little is known about these Anosov flows.

On the other hand, many quasigeodesic flows in hyperbolic 3-manifolds have been constructed, but these are pseudo-Anosov flows. They are generalizations of Anosov flows, where $p$-prong singularities along finitely many closed orbits are allowed. The combination of pseudo-Anosov dynamics and quasigeodesic behavior has powerful implications: Cannon and Thurston [8] proved that suspensions of pseudo-Anosov homeomorphisms of surfaces are quasigeodesic pseudo-Anosov flows. This metric property was used in an essential way to understand the limit sets of fibers and yields as one byproduct examples of sphere filling curves [8]. Mosher [28] proved the quasigeodesic behavior for a class of pseudo-Anosov flows transverse to depth one foliations. This in turn was used to compute Thurston norms via dynamics [26], [27].

Our goal is to study the quasigeodesic property for Anosov flows in hyperbolic manifolds and more generally in 3-manifolds $M$ with negatively curved fundamental group. The universal cover $\widetilde{M}$ of $M$ is homeomorphic to $\mathbf{R}^{3}$ and is compactified with a sphere at infinity $\left(S_{\infty}^{2}=\partial \widetilde{M}\right)$. Associated to the Anosov flow there are two-dimensional foliations, stable $\left(\mathscr{F}^{s}\right)$ and unstable $\left(\mathscr{F}^{u}\right)$, which lift to foliations by topological planes $\widetilde{F}^{s}$ and $\widetilde{F}^{u}$ in $\widetilde{M}$. Consider the following properties: (1) $\mathscr{F}^{s}, \mathscr{F}^{u}$ are quasi-isometric foliations. This means that up to a bounded multiplicative distortion, leaves of $\widetilde{\mathscr{F}}^{s}, \widetilde{\mathscr{F}}^{u}$ are efficient in measuring distance in $\widetilde{M}$; (2) leaves of $\widetilde{\mathscr{F}}^{s}, \widetilde{\mathscr{F}}^{u}$ extend continuously to $S_{\infty}^{2}$, giving continuous parametrizations of their limit sets. Property (1) can actually be defined for any foliation in any manifold, while (2) can be defined whenever the fundamental group $\pi_{1}(M)$ is negatively curved and the leaves of the lifted foliation have well defined intrinsic ideal boundaries. Both of these 
properties are fundamental for understanding the interaction between the foliation structure and the geometry in the large of the manifold. For an Anosov flow $\Phi$ in $M^{3}$ with $\pi_{1}(M)$ negatively curved, (1) is stronger than $\Phi$ being quasigeodesic, which in turn is stronger than (2). In [11] we proved that (1) is never true for a codimension-one foliation in $M^{3}$ with $\pi_{1}(M)$ negatively curved. On the other hand, the continuous extension property holds for fibrations [8] and many depth one foliations [12]. Hence another reason to analyse the quasigeodesic behavior of such flows.

We now describe our results, which show that if $\Phi$ is an Anosov flow in $M^{3}$ with $\pi_{1}(M)$ negatively curved, then the quasigeodesic property is very strong, in the sense that it has several nontrivial consequences for the dynamics of the flow and the limit sets of the leaves. We say that a foliation in $M$ is $\mathbf{R}$-covered if the lift to $\widetilde{M}$ is a foliation whose leaf space is homeomorphic to the set of real numbers $\mathbf{R}$. An Anosov flow in $M^{3}$ is $\mathbf{R}$-covered if both $\mathscr{F}^{s}$ and $\mathscr{F}^{u}$ are $\mathbf{R}$-covered.

Theorem A. If $\Phi$ is a quasigeodesic Anosov flow in $M^{3}$ with $\pi_{1}(M)$ negatively curved, then both $\mathscr{F}^{s}$ and $\mathscr{F}^{u}$ are not $\mathbf{R}$-covered.

This is in stark contrast with suspensions and geodesic flows, both of which are R-covered.

We say that a leaf $F$ of $\widetilde{\mathscr{F}}^{s}$ (or $\widetilde{\mathscr{F}}^{u}$ ) is periodic if it is left invariant by a nontrivial covering translation $g$ of $\widetilde{M}$, that is, $g(F)=F$.

Let $\mathscr{H}^{s}$ and $\mathscr{H}^{u}$ denote the leaf spaces of $\widetilde{\mathscr{F}}^{s}$ and $\widetilde{\mathscr{F}}^{u}$ respectively. If $\mathscr{F}^{s}$ is not R-covered, then $\mathscr{H}^{s}$ is not Hausdorff. The branching leaves of $\widetilde{\mathscr{F}}^{s}$ correspond to the non-Hausdorff points in $\mathscr{H}^{s}$. Two leaves $F \neq$ $F^{\prime} \in \widetilde{F}^{s}$ form a branching pair if the corresponding points in $\mathscr{H}^{s}$ are not separated from each other. This is equivalent to saying that $F, F^{\prime}$ do not have disjoint saturated neighborhoods in $\widetilde{M}$, where a saturated neighborhood of $\widetilde{F}^{s}$ is an open set of $\widetilde{M}$, which is a union of leaves of $\widetilde{\mathscr{F}}^{s}$. Similarly for $\widetilde{\mathscr{F}}^{u}$.

In general very little is known about branching leaves [23], but in the case of quasigeodesic Anosov flows we prove

Theorem B. Let $\Phi$ be a quasigeodesic Anosov flow in $M^{3}$ with $\pi_{1}(M)$ negatively curved. Let $\left(F, F^{\prime}\right)$ be a pair of branching leaves of $\widetilde{F}^{s}$. Then $F$ and $F^{\prime}$ are periodic and invariant under a common nontrivial covering translation of $\widetilde{M}$. This produces a nontrivial free homotopy between closed orbits of $\Phi$ in $M$. Furthermore, up to covering translations, there are only finitely many pairs of branching leaves.

This is the main tool in deriving the following properties of the limit sets of leaves. 
Theorem C. Let $\Phi$ be a quasigeodesic Anosov flow in $M^{3}$ with $\pi_{1}(M)$ negatively curved. Let $F \in \widetilde{\mathscr{F}}^{s}$. Then the limit set of $F$ is neither a Jordan curve in $S_{\infty}^{2}$, nor the whole sphere.

Quasigeodesic behavior implies the continuous extension property (2). The intrinsic ideal boundary of $F \in \widetilde{\mathscr{F}}^{s}$ is a circle at infinity $S_{\infty}^{1}$, where the ideal points correspond to limit points of flow lines. The continuous extension of $F \subset \widetilde{M}$ to the ideal boundary $\partial \widetilde{M}$ then yields a map $S_{\infty}^{1} \rightarrow$ $S_{\infty}^{2}$. As the limit set of $F$ is not a Jordan curve, there are identifications in the image, which are characterized by

Theorem D. Let $\Phi$ be a quasigeodesic Anosov flow in $M^{3}$ with $\pi_{1}(M)$ negatively curved. Let $F \in \widetilde{\mathscr{F}}^{s}$ and $p, q \in F$, not in the same flow line, so that their flow lines converge in the negative direction to the same ideal point in $S_{\infty}^{2}$. Then the two distinct unstable leaves through $p, q$ are periodic and left invariant by a common nontrivial covering translation.

Again this produces freely homotopic closed orbits of $\Phi$ and proves that only finitely many intrinsic ideal points of $F$ can have the same image in $S_{\infty}^{2}$. In fact we find an upper bound for the number of such points. Theorems B, C, and D have analogous statements for $\mathscr{F}^{u}$. Theorem D is also needed for the proof that limit sets are not Jordan curves in Theorem C.

There are many examples of $\mathbf{R}$-covered Anosov flows in hyperbolic $M^{3}$ [13], which are therefore not quasigeodesic. Still, one can prove that in general there are many uniformly quasigeodesic orbits.

Theorem E. Let $\Phi$ be an Anosov flow in $M^{3}$ with $\pi_{1}(M)$ negatively curved. Then, for big enough $K$, there are uncountably many $K$ quasigeodesic orbits of $\Phi$, infinitely many of which are closed.

It is clear from the above theorems that the study of freely homotopic closed orbits is fundamental for understanding quasigeodesic behavior. A detailed analysis of freely homotopic orbits was then necessary. As it turns out, lifts of free homotopies to the universal cover can only be of a particular type, which is in fact quite simple. We stress that neither transitivity of the flow nor $\pi_{1}(M)$ negatively curved is assumed in the next theorem.

Theorem F. Let $\Phi$ be an Anosov flow in $M^{3}$. Assume that $\mathscr{F}^{s}, \mathscr{F}^{u}$ are transversely orientable. Let $\alpha, \beta$ be indivisible closed orbits of $\Phi$ so that for some powers $n, m \in \mathbf{Z}, \alpha^{n}$ is freely homotopic to $\beta^{m}$. Then $\alpha$ is freely homotopic to either $\beta$ or $\beta^{-1}$.

A more precise statement, involving the extremely useful concept of lozenges is given in $\S 3$. The other important technical tool needed for 
the proof of theorems A, B, C and D is the following: orbits of the flow (in the universals over) which stay a bounded distance from each other (say in forward direction) and are not in the same stable leaf, produce freely homotopic closed orbits of the flow in the manifold. When $\Phi$ is a quasigeodesic Anosov flow in $M^{3}$ with $\pi_{1}(M)$ negatively curved, it is easy to construct such bounded distance pairs of orbits.

The organization of the paper is as follows. Preliminaries are covered in $\S 2$. Section 3 contains the analysis of free homotopies between closed orbits. The nonorientable case is also discussed. Section 4 is the core of the paper, where Theorems A, B, and D are proved. Limit sets of leaves are studied in the following section, and finally in the last section we construct the $K$-quasigeodesic orbits.

\section{Preliminaries}

Let $\Phi_{t}: M \rightarrow M$ be a nonsingular $C^{r}(r \geq 1)$ flow in a closed Riemannian manifold $M$. The flow $\Phi$ is Anosov if there is a continuous decomposition of the tangent bundle $T M$ as a Whitney sum $T M=E^{0} \oplus E^{s} \oplus E^{u}$ of $D \Phi_{t}$ invariant subbundles and there are constants $\mu_{0} \geq 1, \mu_{1}>0$ so that:

(i) $E^{0}$ is one dimensional and tangent to the flow,

(ii) $\left\|D \Phi_{t}(v)\right\| \leq \mu_{0} e^{-\mu_{1} t}\|v\|$ for any $v \in E^{s}, t \geq 0$,

(iii) $\left\|D \Phi_{-t}(v)\right\| \leq \mu_{0} e^{-\mu_{1} t}\|v\|$ for any $v \in E^{u}, t \geq 0$.

We restrict to $M$ of dimension 3. The bundles $E^{s}, E^{u}$ are one dimensional and integrate to one-dimensional foliations $\mathscr{F}^{s s}, \mathscr{F}^{u u}$ called the strong stable and strong unstable foliations of the flow. Furthermore, the bundles $E^{0} \oplus E^{s}$ and $E^{0} \oplus E^{u}$ are also integrable [1] producing twodimensional foliations $\mathscr{F}^{s}, \mathscr{F}^{u}$ which are the stable and unstable foliations of the flow.

The leaves of $\mathscr{F}^{s}, \mathscr{F}^{u}$ are either topological planes, annuli or Möbius bands. The last two correspond exactly to leaves containing closed orbits of $\Phi$. There is at most one closed orbit of $\Phi$ in a leaf $F$ of $\mathscr{F}^{s}$, in which case all other orbits in $F$ are forward asymptotic to it. Similarly for $\mathscr{F}^{u}$.

The flow is transitive if the nonwandering set is the whole manifold. Equivalent definitions are: (1) the union of the periodic orbits of $\Phi$ forms a dense subset of $M$ [1], or (2) every leaf of $\mathscr{F}^{s}$ or $\mathscr{F}^{u}$ is dense [1], [31].

The foliations $\mathscr{F}^{s}, \mathscr{F}^{u}$ are Reebless, so Novikov's theorem [29] implies that no closed transversal to either of them is null homotopic. Also, 
given any closed orbit $\gamma$ of $\Phi, \gamma^{n}$ is not null homotopic for any $n \neq 0$ [1]. Let $\pi: \widetilde{M} \rightarrow M$ be the universal covering space of $M$. This notation will be fixed throughout the article. The Anosov foliations $\mathscr{F}^{s}, \mathscr{F}^{u}$ lift to foliations $\widetilde{\mathscr{F}}^{s}, \widetilde{F}^{u}$ in $\widetilde{M}$. The leaves of $\widetilde{\mathscr{F}}^{s}, \widetilde{\mathscr{F}}^{u}$ are topological planes [29], so $\widetilde{M}$ is homeomorphic to $\mathbf{R}^{3}$ [30]. Therefore $M$ is irreducible, that is, every embedded two-dimensional sphere bounds a 3-ball in $M$.

The induced flow in $\widetilde{M}$ is denoted by $\widetilde{\Phi}$. Let $\mathscr{O}$ be the orbit space of $\widetilde{\Phi}$ obtained by collapsing flow lines to points. A fundamental property which will be repeatedly used is that $\mathscr{O}$ is Hausdorff and therefore homeomorphic to $\mathbf{R}^{2}$ [13]. Then $\widetilde{\mathscr{F}}^{s}, \widetilde{\mathscr{F}}^{u}$ induce two transverse onedimensional foliations in $\mathscr{O}$. This is a simplification of the structure in $\widetilde{M}$ and helps in visualizing many arguments. We will occasionally identify sets in $\widetilde{M}$ or orbits of $\widetilde{\Phi}$ with their respective images in $\mathscr{O}$.

Let $W^{s}(x)$ be the leaf of $\mathscr{F}^{s}$ containing $x$ and similarly define $W^{u}(x)$, $W^{s s}(x), W^{u u}(x), \widetilde{W}^{s}(x), \widetilde{W}^{u}(x), \widetilde{W}^{s s}(x)$ and $\widetilde{W}^{u u}(x)$. General references for Anosov flows are [1], [2], [5]-[7], [32], [33].

A recurring technical problem will be the question of transverse orientability of $\mathscr{F}^{s}$ and $\mathscr{F}^{u}$. It turns out that for most results transverse orientability is inessential, but makes the proofs much simpler. We will lift to a transversely orientable cover whenever possible. Unfortunately some results about freely homotopic orbits are false without transverse orientability.

An incompressible surface in $M^{3}$ is an embedded surface which injects in the fundamental group level. A closed 3-manifold is toroidal if it contains an incompressible torus and atoroidal otherwise.

Franks and Williams [15] and Christy [9] produced many examples of intransitive Anosov flows in dimension 3. It is a known fact that intransitivity implies that the manifold is toroidal. However the only written proof of this was given by Mosher [26], who actually proves this result for the more general case of pseudo-Anosov flows. In this generality, techniques of essential laminations [18] are needed, which is not the case for Anosov flows. Here we give a simpler proof for the Anosov flow case.

Proposition 2.1. Let $\Phi$ be an intransitive Anosov flow in $M^{3}$ orientable. Then $M$ is toroidal.

Proof of 2.1. As $\Phi$ is intransitive, the nonwandering set $\Omega$ is a proper compact subset of $M$. By Smale's spectral decomposition theorem [33], $\Omega$ decomposes into finitely many basic sets, each of which is a compact subset of $M$ on which $\Phi$ is transitive. Since $\Phi$ is intransitive in $M$, there are at least two basic sets, and at least one of them, call it $\mathscr{A}$, is attracting. 
Using Lyapounov functions [10] one finds an isolating neighborhood $N$ of $\mathscr{A}$ with $\Phi$ transverse to $\partial N$. Hence $\mathscr{F}^{s}, \mathscr{F}^{u}$ induce foliations in $\partial N$ and each component of $\partial N$ has zero Euler characteristic. Since $\partial N$ is transverse to $\Phi, \partial N$ is two sided in $M$. But the orientability of $M$ then implies that $\partial N$ has to be orientable; hence $\partial N$ is a union of tori. We show that each torus is incompressible, therefore $M$ is toroidal.

Choose a component $T$ of $\partial N$. If $T$ is not incompressible, there is an immersed disk in $M$ with boundary contained in $T$, not null homotopic in $T$. Using classical techniques of cut and paste [22], [24], this produces an embedded disk $D$ with $D \cap T=\partial D$ and $\partial D$ not null homotopic in $T$. As $M$ is irreducible it follows that either: (1) $T$ bounds a solid torus $E$ or (2) one component $E$ of $M-T$ is contained in a closed ball.

In case (1) assume first that $\mathscr{A} \subset E$. After a small perturbation of $D$ we can assume $D$ is transverse to $\mathscr{F}^{u}$ except for isolated saddle, center, and boundary singularities. Let $\mathscr{F}_{D}^{u}$ be the induced singular foliation in $D$. If some leaf of $\mathscr{F}_{D}^{u}$ is not compact, then by the Poincaré-Bendixson theorem, it limits on a closed curve in $D$. The limit curve has nontrivial holonomy, hence cannot be null homotopic in its leaf. Since $\mathscr{F}^{u}$ is Reebless, this curve is also not null homotopic in $M$, a contradiction to $D$ being a disk.

Let $\beta$ be a closed orbit of $\Phi$ in $\mathscr{A}$. As $\mathscr{A}$ is an attractor, $W^{u}(\beta) \subset$ $\mathscr{A}$, hence the closure of $W^{u}(\beta)$ is contained in the interior of $E$. By the above all components of $W^{u}(\beta) \cap D$ are compact, but since $W^{u}(\beta)$ is not compact there may be infinitely many components. In fact this happens because $W^{u}(\beta)$ is dense in $\mathscr{A}$, so $W^{u}(\beta)$ limits on itself.

Let $\alpha \subset D$ be a simple closed curve which is either contained in a component of $W^{u}(\beta) \cap D$ or is the limit of a sequence of nested curves (with respect to $D$ ) in $W^{u}(\beta) \cap D$. Let $D_{\alpha}$ be the disk which $\alpha$ bounds in $D$. We say that $\alpha$ is maximal if $D_{\alpha}$ is not contained in any other disk with the same properties. Since the interior of any $D_{\alpha}$ contains at least one singularity of $\mathscr{F}_{D}^{u}$ and all maximal disks are disjoint, it follows that there are only finitely many such maximal disks.

By the Reebless property $\alpha$ bounds a disk $F_{\alpha}$ in its unstable leaf. Then $F_{\alpha}$ is compact and $W^{u}(\beta)$ does not limit on the outside (with respect to $D_{\alpha}$ ) of $F_{\alpha}$. So we may assume $D$ is transverse to $F_{\alpha}$, and $F_{\alpha} \cap D$ has finitely many components. Again, using cut and paste arguments, $D$ may be isotoped inside $E$ to as to eliminate all intersections with $W^{u}(\beta)$ inside $D_{\alpha}$ (this uses the fact that $W^{u}(\beta)$ does not limit on one side of $F_{\alpha}$ ). By induction on the number of maximal curves we may assume that 
$D \cap \widetilde{W}^{u}(\beta)=\varnothing$. But then $W^{u}(\beta)$ is contained in a 3-ball and $\beta$ is null homotopic, contradiction.

If $\mathscr{A} \cap E=\varnothing$, choose a repeller $\mathscr{B} \subset E$ and apply the argument above to $\mathscr{B}$. This finishes the proof of case (1).

In case (2) we find either a repeller or an attractor in a closed ball. A periodic orbit would be null homotopic, contradiction. q.e.d.

The following is a simple consequence of the proof.

Corollary 2.2. Let $\Phi$ be an Anosov flow in $M^{3}$. If $T$ is an embedded torus transverse to $\Phi$, then $T$ is incompressible.

Proof of 2.2. The proof of the previous proposition shows that if $T$ is compressible, then either $T$ bounds a solid torus or $T$ is contained in a 3-ball. In particular $T$ is separating and $\Phi$ is intransitive. One can then apply the rest of the proof of the proposition.

In the next result we assume that $\pi_{1}(M)$ is negatively curved, as defined by Gromov [21], who used the term hyperbolic. Examples are fundamental groups of closed manifolds of negative curvature. Recall that $\pi_{1}(M)$ being negatively curved implies that $\mathbf{Z} \oplus \mathbf{Z}$ does not inject in $\pi_{1}(M)$ [21], therefore $M$ is atoroidal. Notice that if $M$ is not orientable, then the lift of $\Phi$ to an orientable double cover is transitive if and only if $\Phi$ is. Therefore the next result follows.

Corollary 2.3. If $\Phi$ is an Anosov flow in a 3-manifold with negatively curved fundamental group, then $\Phi$ is transitive.

\section{Freely homotopic orbits}

It will be useful to establish some notation. Given points $x, y \in \widetilde{M}$, define $x \sim_{s} y$ if $x$ and $y$ are in the same leaf of $\widetilde{\mathscr{F}}^{s}$. Similarly define $\sim_{u}, \sim_{s s}, \sim_{u u}$.

Given closed loops $\alpha, \beta$ in $M$, let $\alpha \simeq \beta$ if they are freely homotopic. If $\alpha, \beta$ closed orbits of $\Phi$, satisfy $\alpha \simeq \beta$ by a nontrivial free homotopy, then coherent lifts of $\alpha$ and $\beta$ to $\widetilde{M}$ show that there is a nontrivial covering translation of $\widetilde{M}$ leaving invariant at least 2 leaves of $\widetilde{\mathscr{F}}^{s}$. Our goal is to get a converse to this fact. A priori we only have the following: if $g$ is a nontrivial covering translation with $g\left(F_{i}\right)=F_{i}, i=0,1$, where $F_{0} \neq F_{1} \in \widetilde{F}^{s}$, then $F_{0}, F_{1}$ are periodic and there are (indivisible) closed orbits $\alpha, \beta$ of $\Phi$ in $\pi\left(F_{0}\right), \pi\left(F_{1}\right)$. Since $\pi_{1}\left(\pi\left(F_{0}\right)\right)$ is generated by $\alpha$ and similarly for $\pi_{1}\left(\pi\left(F_{1}\right)\right)$, then $g\left(F_{i}\right)=F_{i}, i=0,1$, imply that for some $n, m \in \mathbf{Z}, \alpha^{n} \simeq \beta^{m}$. 


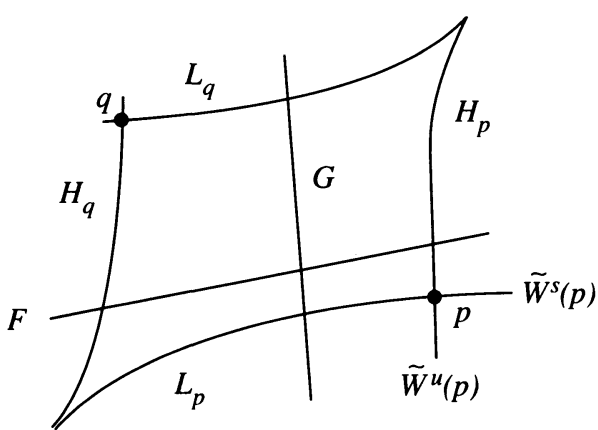

(a)

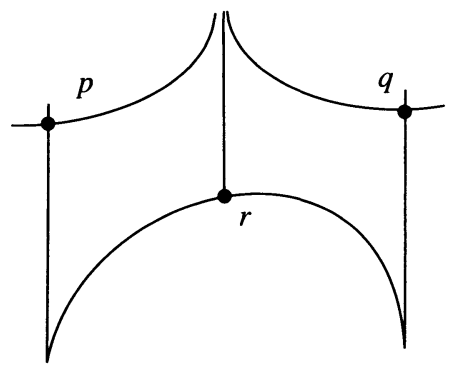

(b)

Figure 1. (a) A lozenge; (b) adjacent or double lozenges

Definition 3.1. Two closed orbits $\alpha, \beta$ of $\Phi$ are almost freely homotopic if there are $n, m \in \mathbf{Z}$ so that $\alpha^{n} \simeq \beta^{m}$.

Suppose $L$ is a simply connected leaf of $\mathscr{F}^{s}, \mathscr{F}^{u}$ or any leaf of $\widetilde{F}^{s}$, $\widetilde{F}^{u}$. Then a half leaf of $L$ is a connected component of $L-\gamma$, where $\gamma$ is any full orbit in $L$. If $L$ is an annulus, a half leaf of $L$ is a connected component of $L-\gamma$ where $\gamma$ is the closed orbit in $L$.

Since $\widetilde{M}$ is simply connected, $\widetilde{\mathscr{F}}^{s}$ and $\widetilde{\mathscr{F}}^{u}$ are always transversely orientable. Choose one such orientation, assumed to agree with the lifts of the transversal orientations to $\mathscr{F}^{s}, \mathscr{F}^{u}$ if any of these is transversely oriented. Notice however that, in general, covering translations will not preserve the transversal orientations.

For $p \in \widetilde{M}$, let $\widetilde{W}_{+}^{s}(p)$ be the half leaf of $\widetilde{W}^{s}(p)$ defined by $\widetilde{\Phi}_{\mathbf{R}}(p)$ and the positive transversal orientation to $\widetilde{F}^{u}$ at $p$. Similarly define $\widetilde{W}_{-}^{s}(p), \widetilde{W}_{+}^{u}(p)$ and $\widetilde{W}_{-}^{u}(p)$. Leaves of $\widetilde{F}^{s}$ and $\widetilde{\mathscr{F}}^{u}$ separate $\widetilde{M}$. If $L \in \widetilde{F}^{s} \cup \widetilde{F}^{u}$, then the front (back) side of $L$ is the component of $\widetilde{M}-L$ defined by the positive (negative) transversal orientation to $L$.

Definition 3.2. Lozenges: Let $p, q \in \widetilde{M}, p \nsim_{s} q, p \nsim_{u} q$. Let $H_{p}$ $\left(L_{p}\right)$ be the half leaf of $\widetilde{W}^{u}(p)\left(\widetilde{W}^{s}(p)\right)$ defined by $\widetilde{\Phi}_{\mathbf{R}}(p)$ and contained in the same side of $\widetilde{W}^{s}(p)\left(\widetilde{W}^{u}(p)\right)$ as $q$. Similarly define $H_{q}, L_{q}$. Then $L_{p}, H_{p}, L_{q}$, and $H_{q}$ form the boundary sides of a lozenge (Figure 1(a))

$$
\forall F \in \widetilde{\mathscr{F}}^{s}, \quad F \cap H_{p} \neq \varnothing \Leftrightarrow F \cap H_{q} \neq \varnothing
$$

and

$$
\forall G \in \widetilde{\mathscr{F}}^{u}, \quad G \cap L_{p} \neq \varnothing \Leftrightarrow G \cap L_{q} \neq \varnothing .
$$

Notice that $\widetilde{W}^{s}(p) \cap \widetilde{W}^{u}(q)=\varnothing$ and $\widetilde{W}^{s}(q) \cap \widetilde{W}^{u}(p)=\varnothing$. The points 
$p, q$ or the orbits $\widetilde{\boldsymbol{\Phi}}_{\mathbf{R}}(p), \widetilde{\boldsymbol{\Phi}}_{\mathbf{R}}(q)$ are called corners of the lozenge. If the lozenge with corner $p$ is contained in the back of $\widetilde{W}^{s}(p)$, then $p$ is a corner of type $(+, *)$, otherwise it is of type $(-, *)$. Similarly, using $\widetilde{W}^{u}(p)$, define types $(*,+),(*,-)$.

Lozenges will be the key tool in the study of freely homotopic orbits and quasigeodesic Anosov flows. By an abuse of notation we will also say that the full leaves $\widetilde{W}^{s}(p), \widetilde{W}^{u}(p), \widetilde{W}^{s}(q)$, and $\widetilde{W}^{u}(q)$ are the sides of the lozenge. Since given any four leaves there is at most one lozenge with sides in them, this is well defined. Two lozenges are adjacent if they share a corner and there is a stable (or unstable) leaf intersecting both interiors (see Figure 1(b)). A sequence of lozenges $\left\{Z_{i}\right\}$ forms a chain if $Z_{i}$ and $Z_{i+1}$ share a corner. Consecutive lozenges may be adjacent or not.

Theorem 3.3. Let $\Phi$ be an Anosov flow in $M^{3}$ so that $\mathscr{F}^{s}$ and $\mathscr{F}^{u}$ are transversely orientable. Let $\alpha_{0}, \alpha_{1}$ be indivisible closed orbits of $\Phi$ which are almost freely homotopic. Then either

(i) $\alpha_{0} \simeq \alpha_{1}$ or

(ii) $\alpha_{0} \simeq\left(\alpha_{1}\right)^{-1}$.

Furthermore, coherent lifts of $\alpha_{0}, \alpha_{1}$ to $\widetilde{M}$ are connected by a finite chain of lozenges.

Proof of 3.3. Lift $\alpha_{0}$ to an orbit $\gamma_{0} \subset \widetilde{M}$ and lift the free homotopy between powers of $\alpha_{0}$ and $\alpha_{1}$ so that $\alpha_{1}$ is lifted to $\gamma$. The free homotopy means that there is a nontrivial covering translation $g$ of $\widetilde{M}$ (which may not be indivisible) leaving $\gamma_{0}$ and $\gamma$ invariant. We first prove that $\gamma_{0}$ is connected to $\gamma$ by a finite chain of lozenges with corners on lifts of periodic orbits, which are all invariant under $g$. let

Let $F_{0}=\widetilde{W}^{s}\left(\gamma_{0}\right), F=\widetilde{W}^{s}(\gamma), \mathscr{E}_{0}$ be the side of $F_{0}$ containing $F$ and

$$
\mathscr{B}_{0}=\bigcup_{p \in L \in \mathscr{L}_{0}}\{p\}, \quad \text { where } \mathscr{L}_{0}=\left\{L \in \widetilde{\mathscr{F}}^{s} \mid L \cap \widetilde{W}^{u}\left(\gamma_{0}\right) \neq \varnothing, L \subset \mathscr{E}_{0}\right\}
$$

Notice $\mathscr{B}_{0}$ is a subset of $\widetilde{M}$, whereas $\mathscr{L}_{0}$ is a subset of $\mathscr{H}^{s}$.

Since $g$ preserves the transversal orientation to $\widetilde{\mathscr{F}}^{s}, g\left(\mathscr{B}_{0}\right)=\mathscr{B}_{0}$. The set of orbits in $\widetilde{W}^{u}\left(\gamma_{0}\right)$ is homeomorphic to $\mathbf{R}$, and $g$ induces a contraction or expansion in this set. As a result $g$ does not leave invariant any leaf of $\widetilde{F}^{s}$ contained in $\mathscr{B}_{0}$. Therefore $F \notin \mathscr{L}_{0}$. The set $\mathscr{B}_{0}$ is an open, connected, $\widetilde{F}^{s}$ saturated, proper subset of $\widetilde{M}$. Hence its boundary is a nonempty union of leaves of $\widetilde{F}^{s}$. Since $F \cap \mathscr{B}_{0}=\varnothing$, either $F \subset \partial \mathscr{B}_{0}$ or there is unique leaf $F_{1} \subset \partial \mathscr{B}_{0}$ which separates $F$ 
from $\mathscr{B}_{0}$. In the second case notice that since $g\left(\mathscr{B}_{0}\right)=\mathscr{B}_{0}$ we have $g\left(F_{1}\right) \subset \partial \mathscr{B}_{0}$. If $g\left(F_{1}\right) \neq F_{1}$, it follows that $g\left(F_{1}\right)$ does not separate $F_{0}$ from $F$, a contradiction to $g$ leaving both $F$ and $F_{0}$ invariant. In either case one finds $F_{1} \subset \partial \mathscr{B}_{0}$, with $g\left(F_{1}\right)=F_{1}$. Then there is an orbit $\gamma_{1} \subset F_{1}$ with $g\left(\gamma_{1}\right)=\gamma_{1}$, and $\gamma_{1}$ projects to a closed orbit of $\Phi$ which is almost freely homotopic to $\alpha_{0}$. If $F_{1} \neq F$, induction produces $F_{i}$ and $\gamma_{i}, i=1,2, \cdots$.

Claim. For some $i, F_{i}=F$; hence $\gamma_{i}=\gamma$.

Suppose not. Let $\mathscr{C}_{i}$ be the side of $F_{i}$ containing $F_{i-1}$. Then $\partial \mathscr{C}_{i}=F_{i}$ and $\mathscr{C}_{i} \subset \mathscr{C}_{i+1}$. Notice that for all $i, F \not \subset \mathscr{C}_{i}$. So

$$
F \not \subset \mathscr{C}=\bigcup_{i \in \mathbf{N}} \mathscr{C}_{i} \text {. }
$$

The set $\mathscr{C}$ is an open, $\widetilde{F}^{s}$ saturated, proper subset of $\widetilde{M}$. Furthermore, $g(\mathscr{C})=\mathscr{C}$. Using the argument above, there is a unique leaf $L \in \widetilde{\mathscr{F}}^{s}$, $L \subset \partial \mathscr{C}$ which is either $F$ or the unique leaf which separates all $\mathscr{C}_{i}$ from $F$. Therefore $g(L)=L$. Let $\gamma^{*}$ be the unique orbit in $L$ with $g\left(\gamma^{*}\right)=\gamma^{*}$. Since $L \subset \partial \mathscr{C}$, the $F_{i}=\widetilde{W}^{s}\left(\gamma_{i}\right)$ are limiting on $L$, so for $i$ big enough,

$$
\varnothing \neq \widetilde{W}^{u}\left(\gamma^{*}\right) \cap \widetilde{W}^{s}\left(\gamma_{i}\right)=\beta_{i}
$$

Since $g$ leaves invariant both of these leaves, $g\left(\beta_{i}\right)=\beta_{i}$, a contradiction to $g\left(\gamma^{*}\right)=\gamma^{*}$ (else there would be two closed orbits in $W^{u}\left(\pi\left(\gamma^{*}\right)\right)$ ). This proves the claim.

Notice that as $\widetilde{W}^{s}\left(\gamma_{1}\right) \subset \partial \mathscr{B}_{0}$, it follows that there is a stable leaf $P$ with

$$
P \cap \widetilde{W}^{u}\left(\gamma_{0}\right) \neq \varnothing \text { and } P \cap \widetilde{W}^{u}\left(\gamma_{1}\right) \neq \varnothing .
$$

Given this we will now show that $\gamma_{0}$ and $\gamma_{1}$ are connected by a chain of adjacent lozenges, all of which intersect a common stable leaf.

By changing the transversal orientations to $\widetilde{\mathscr{F}}^{s}, \widetilde{\mathscr{F}}^{u}$ if necessary assume $\gamma_{1}$ is in the front of $\widetilde{W}^{s}\left(\gamma_{0}\right)$ and in the back of $\widetilde{W}^{u}\left(\gamma_{0}\right)$. Let

$$
\mathscr{D}_{0}=\bigcup_{p \in S \in \mathscr{R}_{0}}\{p\}, \quad \text { where } \mathscr{R}_{0}=\left\{S \in \widetilde{\mathscr{F}}^{u} \mid S \cap \widetilde{W}_{-}^{s}\left(\gamma_{0}\right) \neq \varnothing\right\} \text {. }
$$

Then $g\left(\mathscr{D}_{0}\right)=\mathscr{D}_{0}$ and $\widetilde{W}^{u}\left(\gamma_{1}\right) \cap \mathscr{D}_{0}=\varnothing$. As before there is a unique leaf $H_{1} \subset \partial \mathscr{D}_{0}$ separating $\widetilde{W}^{u}\left(\gamma_{0}\right)$ from $\widetilde{W}^{u}\left(\gamma_{1}\right)$ (or $H_{1}=\widetilde{W}^{u}\left(\gamma_{1}\right)$ ). Furthermore $g\left(H_{1}\right)=H_{1}$. Let $\delta_{1}$ be the unique orbit in $H_{1}$ with $g\left(\delta_{1}\right)=$ $\delta_{1}$. Notice $\widetilde{W}^{u}\left(\delta_{1}\right) \cap \widetilde{W}^{s}\left(\gamma_{0}\right)=\varnothing$, which implies that $\delta_{1}$ is in the front of $\widetilde{W}^{s}\left(\gamma_{0}\right)$. 
Lemma 3.4. $\widetilde{W}_{-}^{u}\left(\delta_{1}\right), \widetilde{W}_{+}^{s}\left(\delta_{1}\right), \widetilde{W}_{+}^{u}\left(\gamma_{0}\right)$, and $\widetilde{W}_{-}^{s}\left(\gamma_{0}\right)$ form a lozenge. Proof of 3.4. Notice that as $\widetilde{W}^{u}\left(\delta_{1}\right) \subset \partial \mathscr{D}_{0}$, there is a $S \in \widetilde{F}^{u}$, with

$$
a \in S \cap \widetilde{W}_{-}^{s}\left(\gamma_{0}\right) \neq \varnothing \text { and } b \in S \cap \widetilde{W}^{s}\left(\delta_{1}\right) \neq \varnothing .
$$

We first claim that $\gamma_{0}$ is in the back of $\widetilde{W}^{s}\left(\delta_{1}\right)$. The transverse orientation to $\widetilde{\mathscr{F}}^{s}$ induces an orientation to $S$ (as a subset of $\mathscr{O}$ ). If $\gamma_{0}$ were in the front of $\widetilde{W}^{s}\left(\delta_{1}\right)$, then orientations of $S$ in $a, b$ would be contradictory (see Figure 2(a)).

Suppose now that $S$ intersects $\widetilde{W}_{-}^{s}\left(\delta_{1}\right)$ (see Figure 2(b)). By taking inverse if necessary, assume that $g$ induces a contraction on the set of orbits of $\widetilde{\Phi}$ in $\widetilde{W}^{s}\left(\gamma_{0}\right)$. Since $S$ separates $\widetilde{M}$, it follows that $g$ induces a contraction on the set of orbits in $\widetilde{W}^{s}\left(\delta_{1}\right)$ also. Then $g$ induces expansions on the sets of orbits in $\widetilde{W}^{u}\left(\gamma_{0}\right)$ and $\widetilde{W}^{u}\left(\delta_{1}\right)$. As a result there is $L \in \widetilde{\mathscr{F}}^{s}, L \cap S \neq \varnothing$ which is invariant under $g$ and with $L \subset \partial \mathscr{B}_{0}$. But then $L$ separates $\widetilde{W}^{u}\left(\delta_{1}\right)$ from $\gamma_{0}$, hence also separates $\widetilde{W}^{u}\left(\gamma_{1}\right)$ from $\gamma_{0}$, and therefore $L$ separates $\widetilde{W}^{s}\left(\gamma_{1}\right)$ from $\gamma_{0}$. But since $\widetilde{W}^{s}\left(\gamma_{1}\right) \subset \partial \mathscr{B}_{0}$, the only stable leaves which possibly separate $\widetilde{W}^{s}\left(\gamma_{1}\right)$ from $\gamma_{0}$ are contained in $\mathscr{B}_{0}$. Therefore $L \subset \mathscr{B}_{0}$. As seen before there is no stable leaf contained in $\mathscr{B}_{0}$ which is invariant under $g$. This is a contradiction to $g(L)=L$. We conclude that $S$ intersects $\widetilde{W}_{+}^{s}\left(\delta_{1}\right)$.

Using $(*)$ above, the $g$ invariance of the sets $\widetilde{W}_{+}^{s}\left(\delta_{1}\right), \widetilde{W}_{-}^{s}\left(\gamma_{0}\right)$, and $\mathscr{D}_{0}$; and the fact that $\mathscr{D}_{0}$ is connected it now follows that

$$
\left\{H \in \widetilde{F}^{u} \mid H \cap \widetilde{W}_{-}^{s}\left(\gamma_{0}\right) \neq \varnothing\right\}=\left\{H \in \widetilde{\mathscr{F}}^{u} \mid H \cap \widetilde{W}_{+}^{s}\left(\delta_{1}\right) \neq \varnothing\right\} .
$$

Since $\gamma_{1} \subset \partial \mathscr{B}_{0}$, there is $F^{\prime} \in \widetilde{\mathscr{F}}^{s}$ intersecting $\widetilde{W}_{+}^{u}\left(\gamma_{0}\right)$ and $\widetilde{W}^{u}\left(\gamma_{1}\right)$. If $\gamma_{1} \neq \delta_{1}$, recall that $\widetilde{W}^{u}\left(\delta_{1}\right)$ then separates $\widetilde{W}^{u}\left(\gamma_{1}\right)$ from $\widetilde{W}^{u}\left(\gamma_{0}\right)$. In any case it thus follows that $F^{\prime} \cap \widetilde{W}^{u}\left(\delta_{1}\right) \neq \varnothing$. But $\gamma_{0}$ in the back of $\widetilde{W}^{s}\left(\delta_{1}\right)$ implies $F^{\prime} \cap \widetilde{W}_{-}^{u}\left(\delta_{1}\right) \neq \varnothing$. Then as above

$$
\left\{E \in \widetilde{F}^{s} \mid E \cap \widetilde{W}_{+}^{u}\left(\gamma_{0}\right) \neq \varnothing\right\}=\left\{E \in \widetilde{F}^{s} \mid E \cap \widetilde{W}_{-}^{u}\left(\delta_{1}\right) \neq \varnothing\right\} .
$$

This together with $(* *)$ shows that $\gamma_{0}$ and $\delta_{1}$ are the corners of a lozenge, and finishes the proof of the lemma.

If $H_{1} \cap F_{1} \neq \varnothing$, then $H_{1} \cap F_{1}=\delta_{1}$ is the periodic orbit in $F_{1}$, so $\delta_{1}=\gamma_{1}$ and we are done. Otherwise start with $\delta_{1}, \gamma_{1}$ and proceed as above. As before this procedure can only be repeated finitely many times. Therefore $\gamma_{0}$ is connected to $\gamma_{1}$ by a finite chain of lozenges. In addition 


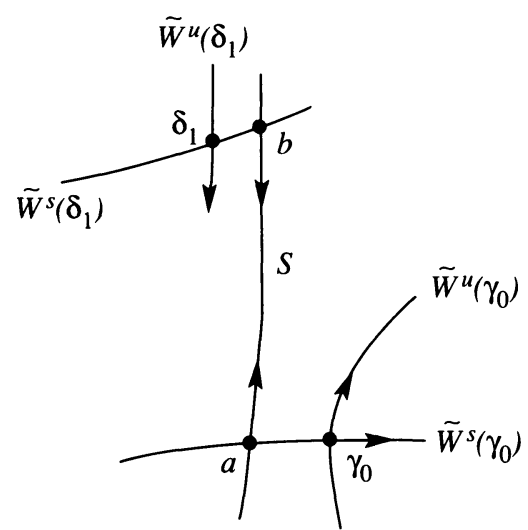

(a)

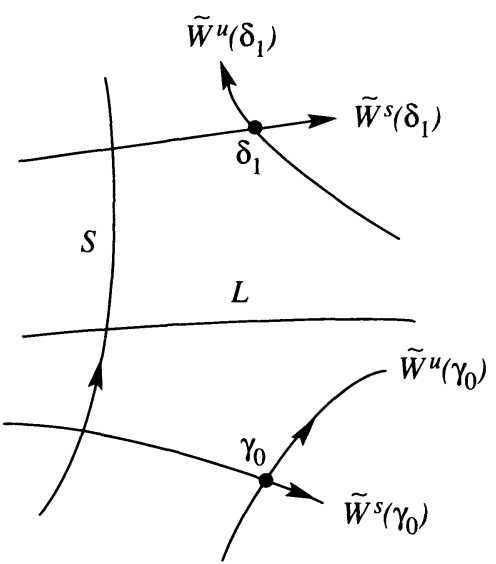

(b)

FIGURE 2. (a) Eliminating $\gamma_{0}$ in the front of $\widetilde{W}^{s}\left(\delta_{1}\right)$; (b) eliminating $\gamma_{0}$ in the back of $\widetilde{W}^{u}\left(\delta_{1}\right)$. The arrows indicate positive orientations in leaves of $\widetilde{F}^{s}$ (or $\widetilde{F}^{u}$ ) as subsets of $\mathscr{O}$. These orientations are induced by the transversal orientations to the dual foliation.

all lozenges in this chain are adjacent and intersect a common stable leaf, as desired.

In the same way $\gamma_{i}$ and $\gamma_{i+1}$ are connected by a chain of lozenges. By induction it follows that $\gamma_{0}$ and $\gamma$ are connected by a finite chain of lozenges. All lozenges and their corners are left invariant by $g$.

Finally, let $\varepsilon_{0}, \varepsilon_{1}$ be corners of a lozenge in $\widetilde{M}$. Let $\beta_{i}=\pi\left(\varepsilon_{i}\right)$ and assume that $\beta_{0}$ is a closed orbit of $\Phi$. Let now $f$ be the covering translation associated to the indivisible orbit $\beta_{0}$, so that $f\left(\varepsilon_{0}\right)=\varepsilon_{0}$. Since $f$ preserves transversal orientations, it follows that $f$ preserves the sets corresponding to $\mathscr{D}_{0}$ and $\mathscr{B}_{0}$ in the above construction, so it preserves their intersection which is exactly the lozenge. Therefore $f\left(\varepsilon_{1}\right)=\varepsilon_{1}$. This implies that $\beta_{0} \simeq \beta_{1}^{n}$ for some $n \in \mathbf{Z}$. Similarly $\beta_{1} \simeq \beta_{0}^{m}$, which implies $n m=1$, so either $n=m=1$ or $n=m=-1$. In fact the action of $f$ on $\widetilde{W}^{u}\left(\varepsilon_{i}\right)$ shows that $f$ is an expansion in one of them and a contraction in the other, therefore $\beta_{0} \simeq\left(\beta_{1}\right)^{-1}$. Induction then finishes the proof of the theorem.

We in fact also proved the following result which is an extremely useful technical tool. Transitivity of the flow is not assumed. 
Theorem 3.5. Let $\Phi$ be an Anosov flow in $M^{3}$. Let $F_{0} \neq F_{1}$ be leaves of $\widetilde{\mathscr{F}}^{*}$ for which there is a nontrivial covering translation $g$ with $g\left(F_{i}\right)=F_{i}, i=0,1$. Let $\gamma_{i} \subset F_{i}$ be the orbits of $\widetilde{\Phi}$ with $g\left(\gamma_{i}\right)=\gamma_{i}$. Then $\gamma_{0}$ and $\gamma_{1}$ are connected by a finite chain of lozenges. All lozenges in the chain are left invariant by $g$ and so are the respective corners.

Notice that it follows from the hypothesis of the theorem that $g$ preserves transversal orientations to $\widetilde{\mathscr{F}}^{s}$ and $\widetilde{\mathscr{F}}^{u}$.

Remarks. (1) When considering almost freely homotopic orbits, the case $\alpha \simeq \beta^{-1}$ is quite common. It occurs for instance in geodesic flows. If $\alpha$ represents an oriented closed geodesic in an orientable surface of negative curvature and $\beta$ is the same geodesic with the reversed orientation, then in the unit tangent bundle $\alpha \simeq \beta^{-1}$.

(2) When $\mathscr{F}^{s}, \mathscr{F}^{u}$ are not transversely orientable, Theorem 3.3 may be false. Consider the geodesic flow in $T_{1} N^{2}, N$ of negative curvature, nonorientable. Let $\alpha$ be an orientation reversing closed geodesic in $N$ and fix an orientation in $\alpha$. Let $\beta$ be the same geodesic being traversed in the opposite orientation. Then $\alpha^{2} \simeq \beta^{-2}$ by just turning the angle along $\alpha^{2}$ by $\pi$. But $\alpha$ is not freely homotopic to $\beta$. One cannot consistently turn the angle along $\alpha$ because $\alpha$ is not "transversely" orientable.

Corollary 3.6. Let $\Phi$ be an Anosov flow in $M$, and $\alpha_{0}, \alpha_{1}$ indivisible, almost freely homotopic closed orbits of $\Phi$. Then there are integers $i, j \in$ $\{1,-1,2,-2\}$ so that $\alpha_{0}^{i} \simeq \alpha_{1}^{j}$.

Proof of 3.6. Consider the transversal orientations to $\widetilde{F}^{s}, \widetilde{F}^{u}$ in $\widetilde{M}$. A covering translation $g$ either fixes the transversal orientation to $\widetilde{\mathscr{F}}^{s} \mathrm{ev}$ erywhere or reverses it everywhere. The same happens to $\widetilde{\mathscr{F}}^{u}$. Therefore $g^{2}$ preserves both transversal orientations.

Lift $\alpha_{0}$ to $\gamma_{0}$ in $\widetilde{M}$ and lift $\alpha_{1}$ to $\gamma_{1}$ corresponding to the free homotopy. Let $g$ be the covering translation associated to $\alpha_{0}$, and $h$ be the corresponding covering translation associated to $\alpha_{1}$. Then $g^{n}=h^{m}$ for some $n, m \in \mathbf{Z}$. This implies that $g^{n}\left(\gamma_{0}\right)=\gamma_{0}$ and $g^{n}\left(\gamma_{1}\right)=h^{m}\left(\gamma_{1}\right)=\gamma_{1}$. By Theorem 3.5, $\gamma_{0}$ and $\gamma_{1}$ are connected by a finite chain of connecting lozenges in $\widetilde{M}$. Since $g^{2}$ preserves transversal orientations, the last paragraph of the proof of Theorem 3.3 shows that $g^{2}$ leaves invariant all corners of the lozenges in the chain and therefore $g^{2}\left(\gamma_{1}\right)=\gamma_{1}$. This implies that $\alpha_{0}^{2} \simeq \alpha_{1}^{i}$, for some $i \in \mathbf{Z}$. Similarly $\alpha_{1}^{2} \simeq \alpha_{0}^{j}$; therefore $\alpha_{0}^{4} \simeq \alpha_{0}^{i j}$, giving the possibilities in the result. q.e.d.

We conjecture that $\alpha_{0}^{i} \simeq \alpha_{1}^{j}$ cannot happen for $|i j|=2$. For example $\alpha_{0}^{2} \simeq \alpha_{1}$ should be impossible for indivisible closed orbits. 


\section{Quasigeodesic Anosov flows}

First we analyse a condition which implies the existence of freely homotopic orbits of an Anosov flow. We use the following classical result:

Theorem 4.1 (Closing lemma for Anosov flows) [1], [5]. Let $\Phi$ be an Anosov flow in $M$ closed. Then for any $\theta>0$ there are $\zeta>0$ and $\nu>0$ for which the following is true: if $d\left(\Phi_{t}(x), x\right)<\zeta$ and $t \geq \nu$, then there are $y \in M$ and $t^{\prime} \in \mathbf{R}$ so that

(i) $\Phi_{t^{\prime}}(y)=y$,

(ii) $\left|t-t^{\prime}\right|<\theta$ and

(iii) $d\left(\Phi_{r}(x), \Phi_{r}(y)\right)<\theta$ for $0 \leq r \leq t$.

This means that the almost closed flow segment $\Phi_{s}(x), 0 \leq s \leq t$, is shadowed very near by an actual closed orbit.

Recall that the injectivity radius of $M$, denoted by $\operatorname{inj}(M)$, is defined to be half of the infimum of the lengths of homotopically nontrivial closed loops in $M$. If $M$ is closed, then $\operatorname{inj}(M)>0$. Let $B_{a}(x)$ be those $y \in M$ (or $\widetilde{M}$ ) at most $a$ distant from $x$. The next result is the starting point of the study of quasigeodesic Anosov flows. It will be used to prove most of the results in this section.

We say that two sets in a metric space are a bounded distance apart if there is $R>0$ so that each set is contained in the $R$ distance neighborhood of the other one.

Proposition 4.2. Let $\Phi$ be a transitive Anosov flow in $M^{3}$, and $z_{0}, z_{1}$ points in $\widetilde{M}$ so that

(i) $\widetilde{\Phi}_{[0,+\infty)}\left(z_{0}\right)$ and $\widetilde{\Phi}_{[0,+\infty)}\left(z_{1}\right)$ are a bounded distance apart and

(ii) $z_{0}$ and $z_{1}$ are not in the same leaf of $\widetilde{\mathscr{F}}^{s}$.

Then there are freely homotopic closed orbits of $\Phi$ in $M$. Analogous result holds in the negative direction.

Proof of 4.2. By hypothesis there is $R>0$ so that

$$
\forall t \geq 0, \exists \psi(t) \geq 0, d\left(\widetilde{\Phi}_{t}\left(z_{0}\right), \widetilde{\Phi}_{\psi(t)}\left(z_{1}\right)\right)<R .
$$

Let $\gamma_{t}$ be a geodesic segment in $\widetilde{M}$ of length $<R$ connecting the two points above. Let $w_{m}=\pi\left(z_{m}\right), m=0,1$.

Flow lines in $\widetilde{M}$ are properly embedded. Otherwise using the closing lemma one produces a closed orbit of $\Phi$ which is null homotopic, contradiction. Therefore as

$$
\lim _{t \rightarrow+\infty} \tilde{\Phi}_{t}\left(z_{0}\right)=\infty, \quad \text { then } \lim _{t \rightarrow+\infty} \psi(t)=+\infty
$$




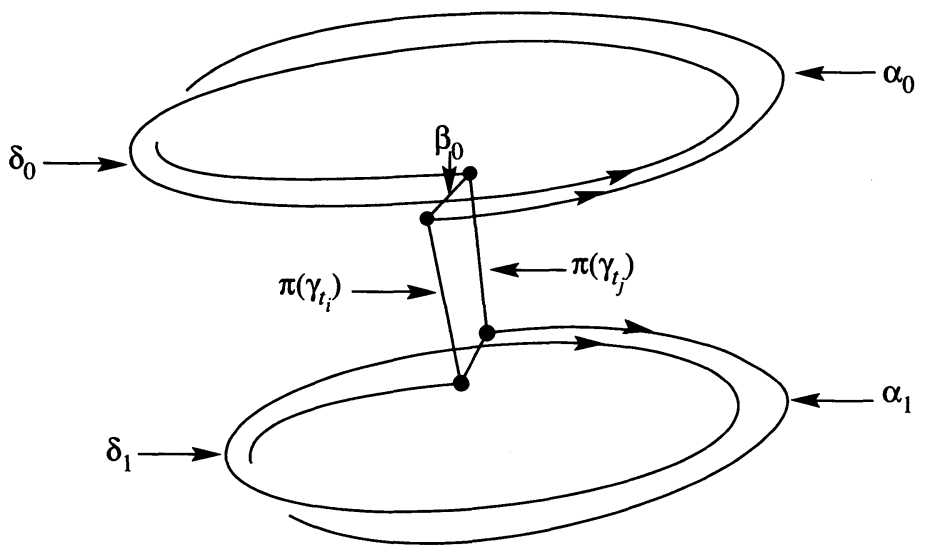

FIGURE 3. Producing freely homotopic orbits.

Now fix $0<\theta<\frac{1}{3} \operatorname{inj}(M)$ and let $\zeta<\frac{1}{3} \operatorname{inj}(M)$ and $\nu$ as given by the closing lemma. Since $M$ is compact and $\gamma_{t}$ is a geodesic arc of bounded length, we can choose a subsequence $t_{i} \rightarrow+\infty$ so that $\Phi_{t_{i}}\left(w_{0}\right)$ converges in $M$ and $\pi\left(\gamma_{t_{i}}\right)$ also converges. Let $i, j$ with $t_{j}-t_{i}>\nu$ and $d\left(\Phi_{t_{i}}\left(w_{0}\right), \Phi_{t_{j}}\left(w_{0}\right)\right)<\zeta$, so that $\pi\left(\gamma_{t_{i}}\right)$ and $\pi\left(\gamma_{t_{j}}\right)$ are at most $\zeta$ apart (see Figure 3 ). Let

$$
\alpha_{0}=\Phi_{\left[t_{i}, t_{j}\right]}\left(w_{0}\right), \quad \alpha_{1}=\Phi_{\left[\psi\left(t_{i}\right), \psi\left(t_{j}\right)\right]}\left(w_{1}\right) .
$$

Since $\pi\left(\gamma_{t_{i}}\right)$ and $\pi\left(\gamma_{t_{j}}\right)$ are at most $\zeta$ apart, it follows that the endpoints of $\alpha_{1}$ are also $\zeta$ apart, so $\alpha_{1}$ is also an almost closed segment in a flow line. The closing lemma produces closed orbits $\delta_{m}, m=0,1$, of $\Phi$ which are $\theta$ near $\alpha_{m}$. Notice $\delta_{m}$ may not be an indivisible closed orbit. Let $\beta_{0}$ be a geodesic path in $B_{\zeta}\left(\Phi_{t_{i}}\left(w_{0}\right)\right)$, connecting $\Phi_{t_{i}}\left(w_{0}\right)$ and $\Phi_{t_{j}}\left(w_{0}\right)$. The condition on the injectivity radius implies this is well defined in the relative homotopy class. Similarly define $\beta_{1}$. By construction it follows that $\delta_{m}$ is freely homotopic to $\alpha_{m} * \beta_{m}$, where $\alpha_{m} * \beta_{m}$ denotes the path $\alpha_{m}$ followed by the path $\beta_{m}$.

Lemma 4.3. $\alpha_{0} * \beta_{0}$ is freely homotopic to $\alpha_{1} * \beta_{1}$.

Proof of 4.3. The path $\widetilde{\boldsymbol{\Phi}}_{\left[t_{i}, t_{j}\right]}\left(z_{0}\right) * \gamma_{t_{j}} *\left(\widetilde{\boldsymbol{\Phi}}_{\left[\psi\left(t_{i}\right), \psi\left(t_{j}\right)\right.}\left(z_{1}\right)\right)^{-1} * \gamma_{t_{i}}^{-1}$ is closed in $\widetilde{M}$, therefore null homotopic. It projects in $M$ to the path $\alpha_{0} * \pi\left(\gamma_{t_{j}}\right) * \alpha_{1}^{-1} * \pi\left(\gamma_{t_{i}}\right)^{-1}$. Since $\pi\left(\gamma_{t_{i}}\right)$ and $\pi\left(\gamma_{t_{j}}\right)$ are $\theta$ close and $\theta<$ $\frac{1}{3} \operatorname{inj}(M)$, the closed path $\beta_{0} * \pi\left(\gamma_{t_{i}}\right) * \beta_{1}^{-1} * \pi\left(\gamma_{t_{j}}\right)^{-1}$ is also null homotopic in $M$. These facts imply the results. 
Continuation of the proof of Proposition 4.2. As a consequence it follows that the closed orbits $\delta_{0}$ and $\delta_{1}$ are freely homotopic. It may happen a priori that they are the same orbit and the free homotopy is trivial, for instance if the orbits through $z_{0}$ and $z_{1}$ get very close to each other. We show however that if this is always the case, then $z_{0}$ and $z_{1}$ are in the same leaf of $\widetilde{F}^{s}$.

Choose $\theta_{k}=1 / k$. Let $\zeta_{k}<1 / k$ and $\nu_{k}$ be given by the closing lemma. Let now $s_{k} \rightarrow+\infty$, so that $d\left(\Phi_{s_{k}}\left(w_{0}\right), \Phi_{s_{k+1}}\left(w_{0}\right)\right)<\zeta_{k}$ and $\pi\left(\gamma_{s_{k}}\right), \pi\left(\gamma_{s_{k+1}}\right)$ are at most $\zeta_{k}$ apart. If the corresponding closed orbits $\delta_{0_{k}}$ and $\delta_{1_{k}}$ of $\Phi$ are always the same and trivially freely homotopic, this implies that $\widetilde{\Phi}_{\left[s_{k}, s_{k+1}\right]}\left(z_{0}\right), \widetilde{\Phi}_{\left[\psi\left(s_{k}\right), \psi\left(s_{k+1}\right)\right]}\left(z_{1}\right)$ are at most $2 \theta_{k}=2 / k$ apart. Since

$$
\lim _{t \rightarrow+\infty} \psi(t)=+\infty, \quad \text { then } \widetilde{\Phi}_{[0,+\infty)}\left(z_{0}\right), \widetilde{\Phi}_{[0,+\infty)}\left(z_{1}\right)
$$

are asymptotic in the forward direction. By expansiveness [32], [5] this can only happen if they are in the same leaf of $\widetilde{\mathscr{F}}^{s}$, contrary to assumption.

This yields two closed orbits which are nontrivially freely homotopic, and finishes the proof of the proposition.

Definition 4.4. A quasi-isometry is a map $\rho:(Q, d) \rightarrow\left(Q^{\prime}, d^{\prime}\right)$ between metric spaces for which there is $k \geq 1$ so that, for any $x, y \in Q$,

$$
\begin{aligned}
\max (d(\rho(x), \rho(y)), d(x, y))>k & \\
\Rightarrow & \frac{1}{k} d(x, y) \leq d(\rho(x), \rho(y)) \leq k d(x, y) .
\end{aligned}
$$

Then we say that $\rho$ is a $k$-quasi-isometry.

Definition 4.5. A quasigeodesic curve in $Q$ is the projection to $Q$ of the image of a quasi-isometric embedding $\rho: A \rightarrow \widetilde{Q}$, where $A$ is an interval in $\mathbf{R}$, finite or not and $\widetilde{Q}$ is the universal cover of $Q$. The metric in $A$ is induced from arc length in $\widetilde{Q}$. If $Q$ is compact, being quasigeodesic is independent of the choice of smooth metric in $Q$. A flow for which all flow lines are quasigeodesics is a quasigeodesic flow. Once a metric is fixed, we say that $\gamma$ is a $k$-quasigeodesic if it is the image (in $Q)$ of a $k$-quasi-isometry.

Notice that finite length curves are always $k$-quasigeodesics for some $k$ as opposed to what may happen for infinite length curves.

Definition 4.6. A curve $\gamma$ in $Q$ is a $(k, u)$ quasigeodesic if any segment in $\gamma$ of length $\leq u$ is a $k$-quasigeodesic. It is also called a local quasigeodesic. 
We assume from now on that $\pi_{1}\left(M^{3}\right)$ is negatively curved. This was originally defined by Gromov [21], who used the term hyperbolic. Gromov showed that there is a canonical compactification of $\widetilde{M}$ with an ideal boundary $\partial \widetilde{M}$. When $M$ is irreducible (which is always the case here), Bestvina and Mess [3] proved that $\partial \widetilde{M}$ is homeomorphic to a sphere, which will be denoted by $S_{\infty}^{2}$. Furthermore $\widetilde{M} \cup S_{\infty}^{2}$ is homeomorphic to the closed 3-ball [3]. Basic references for negatively curved groups are [21]; [19].

Sullivan [34] proved that the intrinsic geometry of the leaves of $\widetilde{F}^{s}$ and $\widetilde{\mathscr{F}}^{u}$ is negatively curved in the large. This property was also studied by Gromov [21], who again used the term hyperbolic. Then any leaf $L \in \widetilde{\mathscr{F}}^{s} \cup \widetilde{\mathscr{F}}^{u}$ has a canonical compactification with an intrinsic ideal boundary $\partial L$ [21]. We proved in [11] that $\partial L$ is always homeomorphic to a circle, which will be denoted by $S_{\infty}^{1}$, usually without reference to the particular leaf we are considering. We stress that $S_{\infty}^{1}$ will always be the intrinsic ideal boundary of $L$ and not its image $\partial \widetilde{M}$ when the leaf extends continuously to $\partial \widetilde{M}$. Furthermore $S_{\infty}^{1}$ is defined independently of whether $\Phi$ is quasigeodesic or not.

If $L \in \widetilde{\mathscr{F}^{s}}$, then the intrinsic ideal points correspond to the (distinct) negative limit points of flow lines in $L$ and to the common positive point of all flow lines [13]. The intrinsic geometry of $L \in \widetilde{\mathscr{F}}^{s}$ resembles that of the hyperbolic plane $\mathbf{H}^{2}$ where the flow lines correspond to the geodesics in $\mathbf{H}^{2}$ which have a common limit point in the ideal boundary of $\mathbf{H}^{2}$. Analogous results hold for $\widetilde{\mathscr{F}}^{u}$.

Given a bi-infinite $K$-quasigeodesic in $M$, any lift to $\widetilde{M}$ is a bounded distance from a minimal geodesic of $\widetilde{M}$ [21]. The bound depends only on $K$ and how much $\pi_{1}(M)$ is negatively curved. As a result the quasigeodesic has two distinct limit points in $S_{\infty}^{2}$ [21, §7.2]. Infinite quasigeodesic rays also have a well-defined limit point.

Recall that the limit set of a subset $B$ of $\widetilde{M}$ is $\Lambda_{B}=\bar{B} \cap S_{\infty}^{2}$, where the closure is taken in $\widetilde{M} \cup S_{\infty}^{2}$. Usually $B$ will be a leaf of $\widetilde{\mathscr{F}}^{\infty}$ or $\widetilde{\mathscr{F}}^{u}$.

In [13] we proved that $\Phi$ being quasigeodesic in $M^{3}$ with $\pi_{1}(M)$ negatively curved implies the following: if $L$ is a leaf of $\widetilde{F}^{s} \cup \widetilde{F}^{u}$, then the embedding $\phi: L \rightarrow \widetilde{M}$ extends continuously to $\phi: L \cup S_{\infty}^{1} \rightarrow \widetilde{M} \cup S_{\infty}^{2}$. In particular $\Lambda_{L}=\phi\left(S_{\infty}^{1}\right)$, giving continuous parametrization of the limit set of each leaf. We then say that $\widetilde{F}^{s}$ and $\widetilde{\mathscr{F}}^{u}$ have the continuous extension property.

This yields that when $\Phi$ is quasigeodesic, there is a continuous function 


$$
\eta_{+}: \widetilde{M} \rightarrow S_{\infty}^{2}, \quad \eta_{+}(x)=\lim _{t \rightarrow \infty} \widetilde{\Phi}_{t}(x) .
$$

The same notation will be used for the corresponding function $\mathscr{O} \rightarrow S_{\infty}^{2}$. Similarly define $\eta_{-}$. Then $\eta_{+}(x) \neq \eta_{-}(x)$ for any $x \in \widetilde{M}$.

If $p \sim_{s} q$, let $\gamma_{s}(p, q)$ be the path from $p$ to $q$ consisting of a segment in $\widetilde{W}^{s s}(p)$ from $p$ to $\widetilde{\Phi}_{\mathbf{R}}(q)$, followed by a segment in $\widetilde{\Phi}_{\mathbf{R}}(q)$. Similarly define $\gamma_{u}(p, q)$ if $p \sim_{u} q$. We remark that given $\varepsilon>0$, there is a uniform $\delta>0$ such that the lengths of $\gamma_{s}(p, q)$ and $\gamma_{u}(p, q)$ are less than $\varepsilon$ if $d(p, q)<\delta[1]$.

Transitivity (Corollary 2.3) implies that for a quasigeodesic Anosov flow in $M$, there is $K>0$ so that all flow lines are uniformly $K$ quasigeodesics. Then all flow lines (in $\widetilde{M}$ ) are a globally bounded distance from corresponding minimal geodesics.

Given any curve $\alpha$ in $M$ or $\widetilde{M}$ let $l(\alpha)$ be its length.

Theorem 4.7. Let $\Phi$ be an Anosov flow ir $M^{3}$ so that $\pi_{1}(M)$ is negatively curved. If $\Phi$ is quasigeodesic, th $\neq^{-s}$ and $\mathscr{F}^{u}$ are not R-covered.

Proof of 4.7. Suppose $\Phi$ is quasigeodesic and let $F$ be a leaf in $\widetilde{\mathscr{F}}^{s}$. There is a continuous extension of the inclusion $\phi: F \rightarrow \widetilde{M}$ to $\phi$ : $F \cup S_{\infty}^{1} \rightarrow \widetilde{M} \cup S_{\infty}^{2}$.

If $\mathscr{F}^{s}$ is R-covered, then we showed in [11] that $\Lambda_{F}=S_{\infty}^{2}$ for any $F \in \widetilde{\mathscr{F}}^{s}$. This implies that $\phi\left(S_{\infty}^{1}\right)=\Lambda_{F}=S_{\infty}^{2}$, hence $\left.\phi\right|_{S_{\infty}^{1}}$ is not injective. Let $c \neq d \in S_{\infty}^{1}$ with $\phi(c)=\phi(d)$.

If $c$ or $d$ is the intrinsic positive ideal point of $F$, then the flow line in $F$ with ideal points $c, d$ is mapped to a quasigeodesic in $\widetilde{M}$ with same limit in both directions. This is impossible.

Let $p, q \in F$ having $c$ and $d$ as ideal points in $S_{\infty}^{1}$ and assume that $p \sim_{s s} q$. By hypothesis, $\eta_{-}(p)=\eta_{-}(q)$. Let $A$ be the segment in $\widetilde{W}^{s s}(p)$ between $p$ and $q$. Let $p_{*}=\pi(p), q_{*}=\pi(q)$.

Both $\widetilde{\Phi}_{\mathbf{R}}(p)$ and $\widetilde{\Phi}_{\mathbf{R}}(q)$ are a bounded distance from minimal geodesics connecting the ideal points and they have a common ideal point $\phi(c)=$ $\phi(d)$. But any two geodesics sharing an ideal point are a bounded distance from each other in that direction [21]. Hence there is $R>0$ so that $\widetilde{\Phi}_{(-\infty, 0]}(p)$ and $\widetilde{\Phi}_{(-\infty, 0]}(q)$ are at most $R$ distant from each other.

As in Proposition 4.2 choose $t_{i} \rightarrow-\infty$, with $\left|t_{i}-t_{i-1}\right| \rightarrow \infty$ and so that $\Phi_{t_{i}}\left(p_{*}\right)$ converges to $p^{\prime}$ in $M$. Then choose $s_{i}$ with $d\left(\widetilde{\Phi}_{t_{i}}(p), \widetilde{\Phi}_{s_{i}}(q)\right)<$ $R$, and $\gamma_{i}$ geodesic arc and connecting them. By passing to a subsequence assume $\pi\left(\gamma_{i}\right)$ converges, so $\Phi_{s_{i}}\left(q_{*}\right)$ converges to $q^{\prime}$. Assume all $\Phi_{t_{i}}\left(p_{*}\right)$ are very close. 
Then there are covering translations $g_{i}$ of $\widetilde{M}$ so that

$$
u_{i}=g_{i}\left(\widetilde{\Phi}_{t_{i}}(p)\right) \rightarrow u, \quad v_{i}=g_{i}\left(\widetilde{\Phi}_{s_{i}}(q)\right) \rightarrow v \quad \text { as } i \rightarrow \infty,
$$

where $\pi(u)=p^{\prime}, \pi(v)=q^{\prime}$.

If $u \sim_{s} v$, consider $\gamma_{s}(u, v)$. Since $u_{i} \rightarrow u, v_{i} \rightarrow v$, and $u_{i} \sim_{s} v_{i}$, then using the local product structure of $\widetilde{F}^{s}$ it follows that $\gamma_{s}\left(u_{i}, v_{i}\right)$ converges to $\gamma_{s}(u, v)$. Hence $\gamma_{s}\left(u_{i}, v_{i}\right)$ has bounded length. Isometry under $g_{i}^{-1}$ implies that $\gamma_{s}\left(\widetilde{\Phi}_{t_{i}}(p), \widetilde{\Phi}_{s_{i}}(q)\right)$ also has bounded length. But the strong stable part of these paths is $\Phi_{t_{i}}(A)$, whose length is

$$
l\left(\widetilde{\Phi}_{t_{i}}(A)\right) \geq e^{-\mu_{1} t_{i}} l(A) / \mu_{0} .
$$

This is unbounded since $t_{i} \rightarrow-\infty$.

Therefore $u \nsim_{s} v$, hence $\mathscr{F}^{s}$ is not $\mathbf{R}$-covered, contrary to assumption. So in any case this proves that $\mathscr{F}^{s}$ is not R-covered. Similarly for $\mathscr{F}^{u}$.

Remarks. (1) Bonatti and Langevin [4] recently constructed an example of a transitive, non-R-covered Anosov flow in dimension 3. We should stress that in their example the underlying manifold is toroidal; in fact there is a torus transverse to the flow.

(2) There are many examples of R-covered Anosov flows in $M^{3}$ hyperbolic [13]. By the previous result these cannot be quasigeodesic. In [14] the continuous extension property is studied for these flows.

We now bound the number of orbits of $\widetilde{\Phi}$ which can have the same ideal points in $S_{\infty}^{2}$.

Proposition 4.8. Suppose $\Phi$ is a $K$-quasigeodesic Anosov flow in $M^{3}$ with $\pi_{1}(M)$ negatively curved. Then there is $n_{0}$ depending on $K$ and $M$ so that if $\mathscr{G}=\left\{\gamma_{i}, 1 \leq i \leq n\right\}$ is any set of orbits of $\tilde{\Phi}$ sharing positive and negative ideal points, then $n<n_{0}$.

Proof of 4.8. Choose $b>0$ so that for any $x \in \widetilde{M}$, the ball $B_{b}(x)$ is contained in foliated product boxes of $\widetilde{F}^{s}$ and $\widetilde{F}^{u}$. There is $a>0$ so that any $K$-quasigeodesic is at most $a / 2$ distant from a minimal geodesic of $\widetilde{M}$. Since $M$ is closed, we can choose $n_{1}>0$ so that if $n>n_{1}$, then for any $n$ given points in a ball of radius $(a+1)$ in $\widetilde{M}$, there are at least 2 which are less than $b / 2$ apart. Choose now $n_{0}$ so that if $n>n_{0}$, then for any $n$ points in a ball of radius $(a+1)$, there are at least $n_{1}^{2}$ of them which are mutually at most $b / 2$ apart.

Let $\mathscr{G}=\left\{\gamma_{i}, 1 \leq i \leq n\right\}$ be a set of distinct orbits of $\widetilde{\boldsymbol{\Phi}}$, all sharing endpoints and suppose $n>n_{0}$. Let $\gamma$ be a minimal geodesic of $\widetilde{M}$ having these two common endpoints as ideal points. 
Let $p \in \gamma$ and find $p_{i} \in \gamma_{i}$, with $d\left(p, p_{i}\right)<a$. Then there are at least $n_{1}^{2}$ of them which are in a ball of radius $b / 2$. There are at least $n_{1}$ of them, which are all in distinct stable or unstable leaves. Assume they are $p_{i}, 1 \leq i \leq n_{1}$ and are in distinct stable leaves. Choose now $r_{1}=p_{1}$ and $r_{i} \sim_{s} p_{i}$ so that $r_{i} \sim_{u u} r_{j}$ for all $i, j$. Using $d\left(p_{i}, p_{j}\right)<b / 2$ and product neighborhoods, if $b$ is small enough, then $d\left(r_{i}, r_{j}\right)<b$, for any $i, j$. Notice $\eta_{+}\left(r_{i}\right)=\eta_{+}\left(r_{j}\right), \forall i, j$. Let $a^{\prime}>0$ be the minimum of the unstable distances between the $r_{i}$. Choose $t_{0}>0$ so that $e^{\mu_{1} t_{0}} \partial^{\prime} / \mu_{0} \gg 1$. Choose $q \in \gamma$ far enough from $p$ so that if $\widetilde{\Phi}_{t}\left(r_{i}\right) \in B_{a}(q)$ then $t>t_{0}$. By hypothesis, there are $i, j$ with $1 \leq i, j \leq n_{1}$ so that

$$
q_{i}=\tilde{\Phi}_{t_{i}}\left(r_{i}\right), \quad q_{j}=\tilde{\Phi}_{t_{j}}\left(r_{j}\right), \quad d\left(q_{i}, q\right)<a, \quad \text { and } d\left(q_{i}, q_{j}\right)<b / 2 \text {. }
$$

By assumption $q_{i}$ and $q_{j}$ are in a foliated product box $U$ of $\widetilde{\mathscr{F}}^{u}$. Suppose that $q_{i}$ and $q_{j}$ are not in the same local sheet of $\widetilde{F}^{u}$ in $U$. Consider a path from $q_{i}$ to $q_{j}$ in $\widetilde{W}^{u}\left(q_{j}\right)$, followed by a short transversal to $\widetilde{\mathscr{F}}^{u}$ in $U$ from $q_{j}$ to $q_{i}$. This path can be perturbed to a closed transversal path to $\widetilde{F}^{u}$, contradiction to $\mathscr{F}^{u}$ being Reebless [29].

Therefore $q_{i}$ and $q_{j}$ are in the same local sheet of $\widetilde{\mathscr{F}}^{u}$ in $U$. As a result $d_{u}\left(q_{i}, q_{j}\right)$ is very small, so $l\left(\gamma_{u}\left(q_{i}, q_{j}\right)\right)$ is also small. But its unstable length is $\geq e^{\mu_{1} t_{0}} a^{\prime} / \mu_{0} \gg 1$, contradiction. q.e.d.

Notice there was no concern in getting the best $n_{0}$, which in general will be much smaller than the one predicted here.

We now analyze the identification of ideal points in the forward direction. First notice that flow lines in the same leaf $L$ of $\widetilde{\mathscr{F}}^{s}$ will (forward) converge to the same ideal point of $\widetilde{M}$, because they are asymptotic.

Proposition 4.9. Let $\Phi$ be a non-R-covered Anosov flow in $M^{3}$ with $\pi_{1}(M)$ negatively curved. If the leaves of $\widetilde{\mathscr{F}}^{s}$ and $\widetilde{\mathscr{F}}^{u}$ extend continuously to $\widetilde{M} \cup S_{\infty}^{2}$, then there are nontrivial identifications of limits of flow lines, that is, there are $x, y \in \widetilde{M}$, not in the same leaf of $\widetilde{F}^{s}$ so that $\eta_{+}(x)=$ $\eta_{+}(y)$. Furthermore this happens whenever $\widetilde{W}^{s}(x)$ and $\widetilde{W}^{s}(y)$ form a branching pair. Analogously for $\widetilde{F}^{u}$.

Proof of 4.9. By Theorem 3.4 of [13], both $\widetilde{F}^{s}$ and $\widetilde{F}^{u}$ are not Rcovered. Since $\mathscr{F}^{s}$ is not R-covered, there is branching in $\widetilde{F}^{s}$, so there are $x_{v} \sim_{s} y_{v}$, with $x_{v} \rightarrow x, y_{v} \rightarrow y$ as $v \rightarrow 0$ but $x \varkappa_{s} y$. After a small perturbation, we may assume $x_{v} \in \widetilde{W}^{u}(x)$ and $y_{v} \in \widetilde{W}^{u}(y)$. As $x_{v} \sim y_{v}$, it follows that $\eta_{+}\left(x_{v}\right)=\eta_{+}\left(y_{v}\right)$. Since the map $\phi: \widetilde{W}^{u}(x) \rightarrow \widetilde{M}$ has a 
continuous extension to $\widetilde{W}^{u}(x) \cup S_{\infty}^{1} \rightarrow \widetilde{M} \cup S_{\infty}^{2}$ and $x_{v} \rightarrow x$, we have

$$
\lim _{v \rightarrow 0} \eta_{+}\left(x_{v}\right)=\eta_{+}(x)
$$

Similar reasoning applied to $\widetilde{W}^{u}(y)$ and $y_{v} \rightarrow y$ implies the same for $y$. As a result, $\eta_{+}(x)=\eta_{+}(y)$ and $x \chi_{s} y$. This finishes the proof.

This result is also true (except for the last assertion) in the case of an R-covered Anosov flow which has the continuous extension property [14].

Corollary 4.10. If $\Phi$ is quasigeodesic in $M^{3}$ with $\pi_{1}(M)$ negatively curved, then there are nontrivially, freely homotopic closed orbits of $\Phi$.

Proof of 4.10. By Theorem 4.7, $\Phi$ is not R-covered. Since $\Phi$ is quasigeodesic, the leaves of $\widetilde{\mathscr{F}}^{s}$ and $\widetilde{\mathscr{F}}^{u}$ have the continuous extension property [13]. Using the previous proposition, choose $x \chi_{s} y$ with $\eta_{+}(x)=\eta_{+}(y)$. Then $\widetilde{\Phi}_{\mathbf{R}}(x), \widetilde{\Phi}_{\mathbf{R}}(y)$ are quasigeodesics sharing the positive ideal point, so they are a bounded distance apart in the forward direction. The result now follows from Proposition 4.2.

Actually, much more is known. The next theorem gives detailed information about the branching leaves. Let $F_{i}, F \in \widetilde{\mathscr{F}}^{s}$. Then $F_{i} \rightarrow F$ when any unstable segment transversal to $F$ intersects $F_{i}$ for all $i$ large enough.

Theorem 4.11. Let $\Phi$ be a quasigeodesic Anosov flow in $M^{3}$ with $\pi_{1}(M)$ negatively curved. Let $F_{s}, F \neq F^{\prime} \in \widetilde{\mathscr{F}}^{s}$ so that $F_{s} \rightarrow F \cup F^{\prime}$, that is $\left(F, F^{\prime}\right)$ is a branching pair. Then there is a nontrivial covering translation of $\widetilde{M}$ leaving both $F$ and $F^{\prime}$ invariant, and with invariant orbits $\gamma \subset F$ and $\gamma^{\prime} \subset F^{\prime}$. As a result $\pi(\gamma), \pi\left(\gamma^{\prime}\right)$ are almost freely homotopic closed orbits of $\Phi$. Furthermore $\gamma$ and $\gamma^{\prime}$ are connected by an even number of adjacent lozenges.

Proof of 4.11. Assume that $\mathscr{F}^{s}, \mathscr{F}^{u}$ are transversely orientable. Suppose $F, F^{\prime}$ are in the front of $F_{s}$. By Proposition 4.9, for any $x \in F$, $y \in F^{\prime}, \eta_{+}(x)=\eta_{+}(y)$. Since $\widetilde{\Phi}_{\mathbf{R}}(x)$ and $\widetilde{\Phi}_{\mathbf{R}}(y)$ are quasigeodesics in $\widetilde{M}$, it follows, as in Theorem 4.7, that there is $R>0$ so that

$$
d\left(\widetilde{\Phi}_{[0,+\infty)}(x), \widetilde{\Phi}_{[0,+\infty)}(y)\right)<R .
$$

By Proposition 4.2 there are $p, q \in \widetilde{M}$ so that $\gamma=\widetilde{\Phi}_{\mathbf{R}}(p), \gamma^{\prime}=\widetilde{\Phi}_{\mathbf{R}}(q)$ project to almost freely homotopic orbits of $\Phi$. Therefore there is a nontrivial covering translation $g$ with $g\left(\widetilde{W}^{s}(p)\right)=\widetilde{W}^{s}(p)$ and $g\left(\widetilde{W}^{s}(q)\right)=$ $\widetilde{W}^{s}(q)$. Furthermore the proof of Proposition 4.2 implies that $\widetilde{W}^{u}(p) \cap$ $F \neq \varnothing, \widetilde{W}^{u}(q) \cap F^{\prime} \neq \varnothing$.

By Theorem 3.5 there is a finite chain of lozenges $\left\{Z_{i}\right\}_{1 \leq i \leq n}$ in $\widetilde{M}$ connecting $p$ and $q$. By Theorem 3.3, the indivisible closed orbits $\beta_{1}=$ 


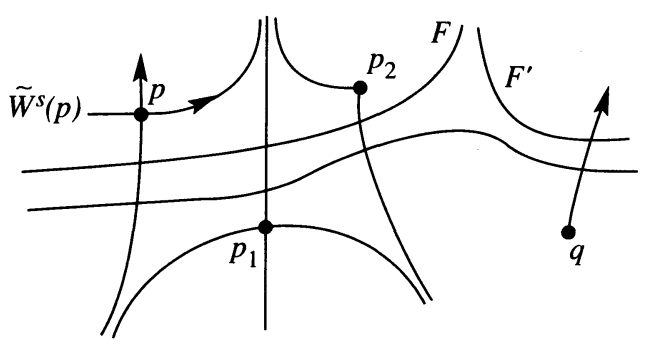

(a)

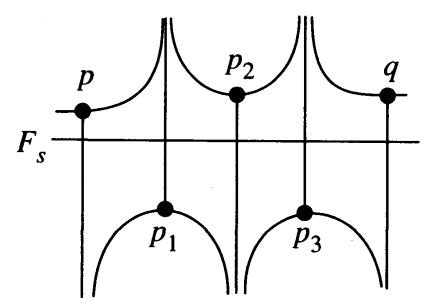

(b)

Figure 4. (a) Rigidity of branching; (b) even chain of lozenges.

$\pi\left(\widetilde{\Phi}_{\mathbf{R}}(p)\right)$ and $\beta_{2}=\pi\left(\widetilde{\Phi}_{\mathbf{R}}(q)\right)$ satisfy $\beta_{1} \simeq \beta_{2}$. This uses the fact that $\mathscr{F}^{s}$ and $\mathscr{F}^{u}$ are transversely orientable. In general, one can only prove that $\beta_{1}^{2} \simeq \beta_{2}^{2}$ which will imply that $(\pi(\gamma))^{2} \simeq\left(\pi\left(\gamma^{\prime}\right)\right)^{2}$. This is the reason for almost freely homotopic in the statement of the theorem.

By changing the transversal orientation to $\widetilde{\mathscr{F}}^{u}$ if necessary, assume $\widetilde{W}^{u}(p)$ is in the back of $\widetilde{W}^{u}(q)$.

We first show that $p \in F, q \in F^{\prime}$. Assume this is not true, say $p \notin F$.

Step 1. Suppose $p$ is in the front of $F$ (Figure 4(a)).

1.a. As $q$ is in the front of $\widetilde{W}^{u}(p)$ and in the back of $\widetilde{W}^{s}(p)$, the proof of Theorem 3.3 shows that $\widetilde{\Phi}_{\mathbf{R}}(p)$ is the $(+,-)$ corner of $Z_{1}$. Let $p_{1}$ be a $(-,+)$ corner of $Z_{1}$. Since $\widetilde{W}_{-}^{u}(p) \cap F \neq \varnothing, \widetilde{W}_{+}^{u}\left(p_{1}\right) \cap F \neq \varnothing$, hence $p_{1}$ is in the back of $F$ and in the back of $\widetilde{W}^{u}(q)$. So clearly $q \notin \widetilde{W}^{u}\left(p_{1}\right)$. Furthermore since $F$ and $F^{\prime}$ are not separated on their negative sides and $F$ is in the front of $\widetilde{W}^{s}\left(p_{1}\right), F^{\prime}$ is also in front of $\widetilde{W}^{s}\left(p_{1}\right)$.

1.b. Suppose $q$ is in the back of $\widetilde{W}^{s}\left(p_{1}\right)$. Since $\widetilde{W}^{u}(q) \cap F^{\prime} \neq \varnothing$ and $F^{\prime}$ is in front of $\widetilde{W}^{s}\left(p_{1}\right), \widetilde{W}^{u}(q) \cap \widetilde{W}^{s}\left(p_{1}\right) \neq \varnothing$. This is a contradiction, because $\widetilde{W}^{u}(q)$ and $\widetilde{W}^{s}\left(p_{1}\right)$ are both invariant under $g$. Hence $q$ is in the front of $\widetilde{W}^{s}\left(p_{1}\right)$.

As $q$ is also in the front of $\widetilde{W}^{u}\left(p_{1}\right)$, it follows that $p_{1}$ is at the $(-,-)$ corner of $Z_{1}$. Choose $p_{2}$ a $(+,+)$ corner of $Z_{2}$. Since $\widetilde{W}_{+}^{u}\left(p_{1}\right) \cap F \neq \varnothing$, $\widetilde{W}_{-}^{u}\left(p_{2}\right) \cap F \neq \varnothing$. Hence $p_{2}$ is in front of $F$ and in particular $q \notin \widetilde{\Phi}_{\mathbf{R}}\left(p_{2}\right)$.

1.c. Induction using 1.a and 1.b shows that corners $p_{i}$ of the lozenges $Z_{i}$ always satisfy $\widetilde{W}^{u}\left(p_{i}\right) \cap F \neq \varnothing$. Hence $\widetilde{\boldsymbol{\Phi}}_{\mathbf{R}}\left(p_{i}\right) \neq \widetilde{\boldsymbol{\Phi}}_{\mathbf{R}}(q)$ for any $i$, contradicting the fact that $\left\{Z_{i}\right\}_{1 \leq i \leq n}$ is a chain from $p$ to $q$. Therefore $p$ cannot be in front of $F$.

Step 2. Suppose $p$ is in the back of $F$. 
As in step 1.b one shows that $Z_{1}$ has $(-,-)$ corner $\widetilde{\Phi}_{\mathbf{R}}(p)$. Let $p_{1}$ be a $(+,+)$ corner of $Z_{1}$. Again as in 1.b, $p_{1}$ is in the front of $F$. Therefore by a similar argument as in step 1, one arrives at a contradiction.

One concludes that $p \in F$ and by symmetry that $q \in F^{\prime}$. Consequently both $F$ and $F^{\prime}$ are invariant under $g$ and hence $\gamma \subset F, \gamma^{\prime} \subset F^{\prime}$.

Step 3. Now redefine $p_{0}=p, p_{n}=q$ and inductively define $p_{i}$, $0<i<n$, to be at the other corner of $Z_{i}$. Let $F_{s}$ near $F$ with $F_{s} \cap$ $\widetilde{W}^{u}(p) \neq \varnothing, F_{s} \cap \widetilde{W}^{u}(q) \neq \varnothing$. If we apply step 1 with $F$ replaced by $F_{s}$ we see that as $p$ is in front of $F_{s}$, (step 1.a) $p_{1}$ is in the back of $F_{s}$ and $\widetilde{W}_{+}^{u}\left(p_{1}\right) \cap F_{s} \neq \varnothing$. By induction $\widetilde{W}^{u}\left(p_{i}\right) \cap F_{s} \neq \varnothing$ for all $i$ and $p_{i}$ is in the front of $F_{s}$ if $i$ is even and in the back of $F_{s}$ otherwise. Since $q$ is in front of $F_{s}$ it follows that $n$ is even, and $F$ and $F^{\prime}$ are connected by an even chain of lozenges. Furthermore all lozenges $Z_{i}$ intersect $F_{s}$. This finishes the proof.

If $\left(F, F^{\prime}\right)$ is a pair of branching leaves which are in the boundary of two adjacent lozenges, then the pivot associated to $F, F^{\prime}$ is the common corner of the lozenges. The pivot always projects to a periodic orbit of $\Phi$ in $M$.

We say that $\left(F, F^{\prime}\right)$ is associated to branching in the positive direction if $F$ and $F^{\prime}$ are not separated on their negative sides.

Theorem 4.7 shows that quasigeodesic behavior forces branching in $\widetilde{\mathscr{F}}^{s}, \widetilde{F}^{u}$. On the other hand, the next result implies these foliations cannot have too much branching.

Proposition 4.12. Let $\Phi$ be a quasigeodesic Anosov flow in $M^{3}$ with $\pi_{1}(M)$ negatively curved. Then, up to covering translations, there are only finitely many branching leaves.

Proof of 4.12. Suppose there are infinitely many inequivalent branching leaves. By changing the flow direction if necessary (this exchanges $\mathscr{F}^{s}$ with $\mathscr{F}^{u}$ ) and maybe also changing the transversal orientation to $\widetilde{F}^{s}$ we may assume there are infinitely many stable branching leaves where the branching occurs in the positive direction. Since $p^{\prime} \in \widetilde{M}$ can be the pivot of at most one pair of adjacent lozenges associated to branching, it follows that there are infinitely many inequivalent pivots. Their images accumulate in $M$, therefore there are pivots $p_{i} \in \widetilde{M}, i \in \mathbf{N}, \widetilde{\Phi}_{\mathbf{R}}\left(p_{i}\right) \neq \widetilde{\Phi}_{\mathbf{R}}\left(p_{k}\right)$ if $i \neq$ $k$ and so that $p_{i} \rightarrow p$ as $i \rightarrow \infty$. By changing the transversal orientation to $\widetilde{F}^{u}$ if necessary assume there are $i, k$ so that $\widetilde{W}_{+}^{s}\left(p_{k}\right) \cap \widetilde{W}_{-}^{u}\left(p_{i}\right) \neq \varnothing$ and $\widetilde{W}_{+}^{u}\left(p_{k}\right) \cap \widetilde{W}_{-}^{s}\left(p_{i}\right) \neq \varnothing$.

For any $n$ let $\Theta_{n}$ be the double lozenge associated to the pivot $p_{n}$. Let $G_{n}, S_{n}$ be the unstable sides of $\Theta_{n}$, so that $G_{n}$ in the back of $S_{n}$, and 


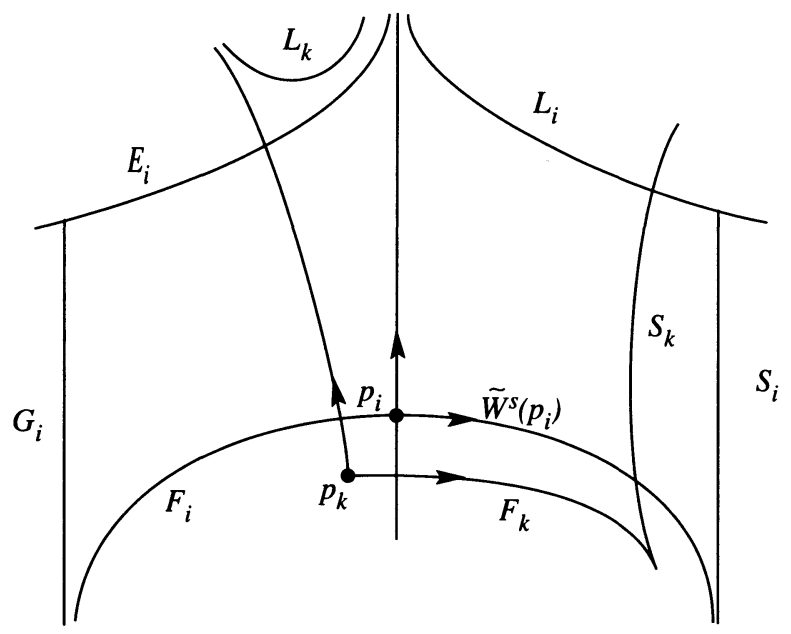

FIGURE 5. Too much branching is unallowed.

let $E_{n}, L_{n}, F_{n}$ be the stable boundaries, with $F_{n}$ in the boundary of the two lozenges in $\Theta_{n}$ and $E_{n}$ in the back of $\widetilde{W}^{u}\left(p_{n}\right)$ (see Figure 5). Then

$$
\widetilde{W}_{+}^{u}\left(p_{k}\right) \cap \widetilde{W}_{-}^{s}\left(p_{i}\right) \neq \varnothing \Rightarrow \widetilde{W}_{+}^{u}\left(p_{k}\right) \cap E_{i} \neq \varnothing .
$$

Therefore $L_{k}$ is in the front of $E_{i}$ and as a result is contained in the back of $\widetilde{W}^{u}\left(p_{i}\right)$ (so $L_{k}$ does not intersect $\widetilde{W}^{u}\left(p_{i}\right)$ ). Similarly $\widetilde{W}_{+}^{s}\left(p_{k}\right) \cap$ $\widetilde{W}_{-}^{u}\left(p_{i}\right) \neq \varnothing$ implies that $S_{k}$ is in the front of $\widetilde{W}^{u}\left(p_{i}\right)$. But this shows that $L_{k} \cap S_{k}=\varnothing$, contradicting the fact that $L_{k}, S_{k}, \widetilde{W}^{s}\left(p_{k}\right), \widetilde{W}^{u}\left(p_{k}\right)$ are the boundary sides of a lozenge with a corner in $p_{k}$. This finishes the proof.

We now analyze the identification of ideal points of stable leaves. Similar results hold for $\widetilde{\mathscr{F}}^{u}$.

Theorem 4.13. Let $\Phi$ be a quasigeodesic Anosov flow in $M^{3}$ closed, with $\pi_{1}(M)$ negatively curved. Let $F \in \widetilde{\mathscr{F}}^{s}$ and $\gamma, \alpha$ be different orbits in $F$, which limit in the same point of $S_{\infty}^{2}$ in the negative direction, that is $\eta_{-}(\gamma)=\eta_{-}(\alpha)$. Then $\widetilde{W}^{u}(\gamma), \widetilde{W}^{u}(\alpha)$ are periodic leaves, and there is a nontrivial covering translation leaving both of them invariant. Furthermore $\widetilde{W}^{u}(\gamma), \widetilde{W}^{u}(\alpha)$ are connected by an even number of adjacent lozenges.

Proof of 4.13. We may assume that $\mathscr{F}^{s}, \mathscr{F}^{u}$ are transitively orientable. Choose $p \in \gamma, q \in \alpha, p \sim_{s s} q$. First notice that $\gamma$ (or $\alpha$ ) cannot be periodic. This is an important property which is true under the much weaker assumption that $\widetilde{W}^{s}(p)$ extends continuously to $S_{\infty}^{2}$. 
Otherwise assume $\gamma$ is periodic. Let $g$ be a nontrivial covering translation with $g(\gamma)=\gamma$. Then

$$
\eta_{-}(\gamma)=\eta_{-}(\alpha) \Rightarrow \eta_{-}\left(g^{n}(\alpha)\right)=g^{n}\left(\eta_{-}(\alpha)\right)=g^{n}\left(\eta_{-}(\gamma)\right)=\eta_{-}(\gamma) .
$$

By replacing $g$ by $g^{-1}$ if necessary, suppose the positive limit point of $\gamma$ is the attracting fixed point of $g$. Then

$$
\lim _{n \rightarrow+\infty} \eta_{-}\left(g^{n}(\alpha)\right)=\eta_{+}(\gamma)
$$

because the intrinsic negative limit points of $g^{n}(\alpha)$ in $F \cup S_{\infty}^{1}$ converge to the intrinsic positive limit point associated to $\gamma$, and $\widetilde{W}^{s}(p)$ extends continuously to $S_{\infty}^{2}$. Thus $\eta_{-}(p)=\eta_{+}(p)$, contradiction to $p$ being in a periodic orbit (which is always a quasigeodesic anyway). Thus $\gamma$ and $\alpha$ are not periodic.

By moving $p, q$ along their unstable leaves to nearby $p^{\prime} \sim_{s} q^{\prime}$, we further assume $\widetilde{W}^{s}(p)$ is not periodic, because $\eta_{-}\left(p^{\prime}\right)=\eta_{-}(p)=\eta_{-}(q)=$ $\eta_{-}\left(q^{\prime}\right)$.

As before choose $t_{i} \rightarrow-\infty$ so that $\Phi_{t_{i}}(\pi(p)) \rightarrow w_{0}$, and choose $s_{i}$ with $d\left(\widetilde{\Phi}_{t_{i}}(p), \widetilde{\Phi}_{s_{i}}(q)\right)<R$, for some global $R$. Let $\gamma_{t_{i}}$ be the geodesic arcs connecting these points, and assume up to subsequence that $\pi\left(\gamma_{t_{i}}\right)$ converges, so $\Phi_{s_{i}}(\pi(q))$ also converges, and let $w_{1}$ be its limit. Suppose that $t_{i}>t_{j}$ for all $i<j$.

Let $g_{i}$ be covering translations so that $p_{i}=g_{i}\left(\tilde{\Phi}_{t_{i}}(p)\right)$ converges to $z_{0}$ (with $\pi\left(z_{0}\right)=w_{0}$ ), and from the above conditions it follows that $q_{i}=g_{i}\left(\tilde{\Phi}_{s_{i}}(q)\right.$ ) converges to $z_{1}$ (with $\pi\left(z_{1}\right)=w_{1}$ ) (see Figure 6). Then as in Theorem $4.7 z_{0} \chi_{s} z_{1}$ and $F_{n}=\widetilde{W}^{s}\left(z_{n}\right), n=0,1$, form a pair of branching leaves. Furthermore Theorem 4.11 implies that $F_{0}$ and $F_{1}$ are connected by an even chain of lozenges. Assume $F_{0}, F_{1}$ are not separated on their negative sides.

Assume that all $p_{i}$ are very close and that all $\gamma_{t_{i}}$ are also near each other. If $p_{i} \sim_{s} p_{j}$, then they are in the same local sheet of $\widetilde{F}^{s}$ near $z_{0}$, so $\pi\left(p_{i}\right)$ and $\pi\left(p_{j}\right)$ are in the same local sheet of $\mathscr{F}^{s}$ near $w_{0}$. This implies that $W^{s}\left(\pi\left(p_{i}\right)\right)$ contains a periodic orbit of $\Phi$, hence $\widetilde{W}^{s}(p)$ is periodic, contradiction. So $p_{i} \chi_{s} p_{j}$, and we may assume $\widetilde{W}^{s}\left(p_{j}\right)$ is in the front of $\widetilde{W}^{s}\left(p_{i}\right)$ for all $j>i$.

The flow segment from $\pi\left(p_{j}\right)$ to $\pi\left(p_{i}\right) \quad(i<j)$ is almost closed, so by the closing lemma, it is shadowed by a closed orbit $\alpha_{0}$ of $\Phi$ (maybe 


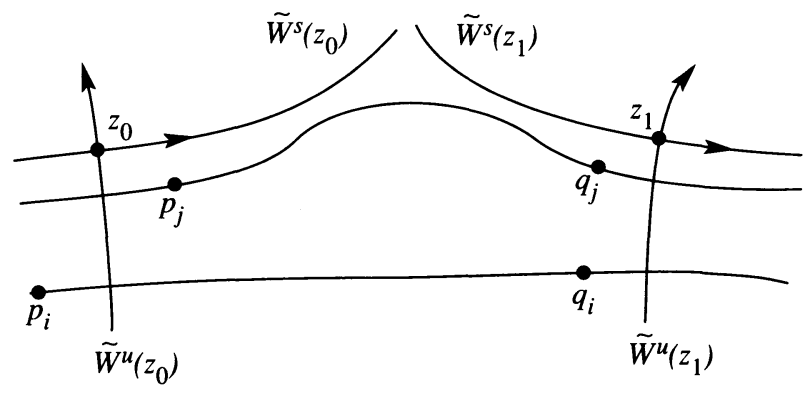

FIGURE 6. Forcing of periodicity in unstable leaves.

$\alpha_{0}$ not indivisible). The same happens to $\pi\left(q_{j}\right), \pi\left(q_{i}\right)$ producing (as in Proposition 4.2) a freely homotopic closed orbit $\alpha_{1}$. Lift $\alpha_{0}$ and $\alpha_{1}$ coherently to $\gamma_{n} \subset \widetilde{M}, n=0,1$, so that $\gamma_{n}$ has a point very near $z_{n}$.

The proof of Theorem 4.11 shows that $\gamma_{n} \subset \widetilde{W}^{s}\left(z_{n}\right), n=1,2$. Since there is only one periodic orbit in $\widetilde{W}^{s}\left(z_{n}\right)$, it follows that the orbits $\gamma_{0}$, $\gamma_{1}$ do not depend on the choice of the pair $i, j$. Furthermore

$$
\lim _{i, j \rightarrow \infty} d\left(p_{i}, p_{j}\right)=0 \Rightarrow \lim _{i \rightarrow \infty} d\left(p_{i}, \gamma_{0}\right)=0
$$

It follows that $\gamma_{0}=\widetilde{\Phi}_{\mathbf{R}}\left(z_{0}\right)$ and similarly $\gamma_{1}=\widetilde{\Phi}_{\mathbf{R}}\left(z_{1}\right)$.

Let $h$ be the covering translation with $h\left(\widetilde{W}^{s}\left(\gamma_{0}\right)\right)=\widetilde{W}^{s}\left(\gamma_{0}\right)$ associated to the indivisible closed orbit $\pi\left(\gamma_{0}\right)$. By taking inverse if necessary assume that $h$ acts as an expansion on the set of orbits in $\widetilde{W}^{s}\left(\gamma_{0}\right)$. As the covering translations taking $\widetilde{\Phi}_{\mathbf{R}}\left(p_{i}\right)$ to $\widetilde{\Phi}_{\mathbf{R}}\left(p_{j}\right)$ leave $\widetilde{W}^{s}\left(\gamma_{0}\right)$ invariant, they are $h^{m_{i j}}$ for some $m_{i j} \in \mathbf{Z}$. Notice that for $i$ fixed:

$$
\lim _{j \rightarrow \infty} h^{m_{i j}}\left(\widetilde{\Phi}_{\mathbf{R}}\left(p_{i}\right)\right)=\widetilde{\Phi}_{\mathbf{R}}\left(z_{0}\right)
$$

Since $p_{n} \notin \widetilde{W}^{s}\left(z_{0}\right)$ for any $n$, the above equation implies that $m_{i j} \rightarrow+\infty$ as $j \rightarrow \infty$ (for $i$ fixed). The only possible way for $h^{m_{i j}}\left(\widetilde{\Phi}_{\mathbf{R}}\left(p_{i}\right)\right)$ not to escape in the stable direction is to have $p_{j} \in \widetilde{W}^{u}\left(\gamma_{0}\right)$ for all $j$ and similarly $q_{j} \in \widetilde{W}^{s}\left(\gamma_{1}\right)$. Therefore $\widetilde{W}^{u}\left(p_{j}\right), \widetilde{W}^{u}\left(q_{j}\right)$ are periodic and left invariant by $h$. In addition $\gamma_{0}$ and $\gamma_{1}$ are connected by an even chain of adjacent lozenges all intersecting a common stable leaf. Translation by $g_{j}^{-1}$ yields the result. 


\section{Limit sets of leaves}

Let $\mathscr{F}$ be a codimension-one foliation in $M^{3}$ with $\pi_{1}(M)$ negatively curved. In general there may be many quasi-isometric leaves. For instance, if $\mathscr{F}$ is a finite depth foliation in $M^{3}$ hyperbolic so that no compact leaf is a fiber of a fibration of $M$ over the circle, then all compact leaves are quasi-isometric [35]. Gabai [17] showed that there are many such foliations. But if there is a dense quasi-isometric leaf, then all leaves are uniformly quasi-isometric and $\mathscr{F}$ is quasi-isometric, contradiction [11]. By Corollary 2.3 all stable and unstable leaves of Anosov flows in such manifolds are dense. Hence:

Corollary 5.1. Let $\Phi$ be an Anosov flow in $M^{3}$ so that $\pi_{1}(M)$ is negatively curved. Then no leaf of $\mathscr{F}^{s}$ or $\mathscr{F}^{u}$ is quasi-isometric.

If there were a quasi-isometric leaf, then its limit set would be a Jordan curve $[21, \S 7.2]$. We prove that not even this weaker property is possible for stable and unstable leaves of quasigeodesic Anosov flows. First we have a preliminary result about limit sets of Reebless foliations.

Proposition 5.2. Let $\mathscr{F}$ be a Reebless foliation in $M^{3}$ with $\pi_{1}(M)$ negatively curved. Let $\mathscr{F}$ be the lift of $\mathscr{F}$ to $\widetilde{M}$ and $F \in \mathscr{F}$. Then $S=S_{\infty}^{2}-\Lambda_{F}$ is a union (which may be empty) of open disks.

Proof of 5.2. Since $\mathscr{F}$ is Reebless, each leaf $F$ of $\mathscr{F}$ is a topological plane. As $F$ is properly embedded (no closed transversals to $\widetilde{F}$ [29]) $\Lambda_{F} \neq \varnothing$. Let $A$ be a component of $S$. Then $A$ is a planar surface, and it suffices to prove it is simply connected.

Let $\gamma$ be a simple closed curve in $A$. Since $\bar{F} \cap \gamma=\varnothing$, there is a neighborhood $V$ of $\gamma$ in $\widetilde{M} \cup S_{\infty}^{2}$ disjoint from $F$. As $\widetilde{M} \cup S_{\infty}^{2}$ is homeomorphic to a closed 3-ball, we may choose $V$ to be homeomorphic to a (closed) solid torus. Cap the solid torus with a closed disk $D \subset \widetilde{M}$ so that $V \cup D$ is simply connected (actually contractible). As $F$ is properly embedded in $\widetilde{M}$, then $F \cap D$ is a compact subset of $F$. Notice that $F \cap \partial D=\varnothing$. Therefore after a small perturbation of $D$ we may assume that $D$ is transverse to $F$. As $\widetilde{M}$ is irreducible and $\mathscr{F}$ is Reebless, classical techniques of cut and paste [22], [24] show $F$ can be isotoped in $\widetilde{M}$ to be disjoint from $D \cup V$. Since $F \cap D$ is compact in $F$, the isotopy can be chosen to be compactly supported, so the limit set of $F$ does not change. This means that $F$ does not limit in one of the components of $S_{\infty}^{2}-V$, therefore $\gamma$ bounds a disk in $A$.

Theorem 5.3. Let $\Phi$ be a quasigeodesic Anosov flow in $M^{3}$ with $\pi_{1}(M)$ negatively curved. Let $F \in \widetilde{\mathscr{F}}^{s}$. Then $\Lambda_{F}$ is not a Jordan curve. 
Proof of 5.3. By lifting to a finite cover if necessary, assume that $\mathscr{F}^{s}$, $\mathscr{F}^{u}$ are transversely orientable. The hypothesis implies that $\mathscr{F}^{s}$ is not R-covered. Therefore the proof of Theorem 4.11 shows that there are two adjacent lozenges both intersecting a common stable leaf. Let $\theta$ be the union of these two lozenges, and let $r, u$, and $s$ be corners of the lozenges, where $u$ is the pivot. Then $\pi\left(\widetilde{\Phi}_{\mathbf{R}}(r)\right) \cong\left(\pi\left(\widetilde{\Phi}_{\mathbf{R}}(u)\right)\right)^{-1} \cong\left(\pi\left(\widetilde{\Phi}_{\mathbf{R}}(s)\right)\right.$ as oriented, indivisible closed orbits of $\Phi$ in $M$. This implies that $\eta_{-}(r)=$ $\eta_{-}(s)$. Since $F$ is dense in $M$, there is a translate $g(F)$ intersecting the unstable boundaries of $\Theta$ in $\widetilde{\Phi}_{\mathbf{R}}(p), \widetilde{\Phi}_{\mathbf{R}}(q)$, where $p \in \widetilde{W}^{u}(r)$ and $q=\widetilde{W}^{u}(s) \cap \widetilde{W}^{s s}(p)$. Thus

$$
\eta_{-}(p)=\eta_{-}(r)=\eta_{-}(s)=\eta_{-}(q) .
$$

This yields that the continuous map $\phi: S_{\infty}^{1} \rightarrow S_{\infty}^{2}$ associated to $g(F)$ is not an embedding. However it is harder to show that its image $\phi\left(S_{\infty}^{1}\right)=$ $\Lambda_{g(F)}$ is not a Jordan curve in $S_{\infty}^{2}$. In order to do that we will need to use Theorems 4.13 and 4.11 .

Suppose then that $J=\phi\left(S_{\infty}^{1}\right)$ is a Jordan curve in $S_{\infty}^{2}$. Assume without loss of generality that $\widetilde{W}^{u}(s)$ is in the front of $\widetilde{W}^{u}(r)$ and also that $\widetilde{W}^{s}(r), \widetilde{W}^{s}(s)$ are not separated on their negative sides, that is, they are associated to positive branching. Let $\Theta_{1}$ and $\Theta_{2}$ be the lozenges whose union is $\Theta$, where $r$ is a $(+,-)$ corner of $\Theta_{1}$. Let $x=\widetilde{W}^{u}(u) \cap \widetilde{W}^{s s}(p)$. Let $a=\eta_{-}(p)$ and $b=\eta_{-}(x)$. Clearly $a \neq b$. Notice that as $p \in$ $\widetilde{W}^{s s}(q)$, then $\gamma_{s}(p, q)$ is the segment in $\widetilde{W}^{s s}(p)$ from $p$ to $q$.

Claim 1. If $v \in \gamma_{s}(p, q)$ and $\eta_{-}(v)=\eta_{-}(p)$, then $v$ is either $p$ or $q$.

If $\eta_{-}(v)=\eta_{-}(p)$ then by Theorem 4.13, $\widetilde{W}^{u}(v)$ is periodic and in addition $\widetilde{W}^{u}(v)$ and $\widetilde{W}^{u}(p)$ are connected by an even chain of lozenges all intersecting $\widetilde{W}^{s}(p)$. Since $v$ is between $\widetilde{W}^{u}(p)$ and $\widetilde{W}^{u}(q)$ and the lozenge has to intersect $\widetilde{W}^{s}(p)$, the first lozenge has to be $\Theta_{1}$. As $v \notin$ $\widetilde{\Phi}_{\mathbf{R}}(u)$, this implies that $v$ is in front of $\widetilde{W}^{u}(u)$. Then the second lozenge of the chain has to be $\Theta_{2}$ with other corner $s$ so $v$ cannot be achieved, contradiction.

A similar argument shows that if $v \in \gamma_{s}(p, q)$ and $\eta_{-}(v)=\eta_{-}(x)$, then $v=x$. Since $J$ is a Jordan curve, this implies that $J_{1}=\eta_{-}\left(\gamma_{s}(p, x)\right)$ is the closure of a component of $J-\{a, b\}$. Similarly for $J_{2}=\eta_{-}\left(\gamma_{s}(x, q)\right)$.

Claim 2. $J_{1}=J_{2}$.

Else $J=J_{1} \cup J_{2}$. But then $\eta_{+}(p)=\eta_{-}(z)$ for some $z \in \gamma_{s}(p, q)$. Then $\eta_{+}(z)=\eta_{+}(p)=\eta_{-}(z)$ contradiction to $\Phi$ being quasigeodesic. Thus the claim is proved. 
Choose now $c \in J_{1}, c \neq a, b$. Then there are $p_{1} \in \gamma_{s}(p, x)$ and $q_{1} \in \gamma_{s}(x, q)$ with $\eta_{-}\left(p_{1}\right)=\eta_{-}\left(q_{1}\right)=c$. Furthermore neither $p_{1}$ nor $q_{1}$ is equal to any of $p, q$ or $x$.

Again by Theorem $4.13, \widetilde{W}^{u}\left(p_{1}\right)$ and $\widetilde{W}^{u}\left(q_{1}\right)$ are periodic and connected by an even chain of lozenges all intersecting $\widetilde{W}^{s}(p)$. Let $\alpha_{0}$ be the periodic orbit in $\widetilde{W}^{u}\left(p_{1}\right)$. If any corner $\alpha^{*}$ of the chain is contained in $\Theta$, then since $\Theta$ is a union of adjacent lozenges, it follows that $\widetilde{W}^{s}\left(\alpha^{*}\right) \cap \widetilde{W}^{u}\left(q_{1}\right) \neq \varnothing$. This is a contradiction to both being left invariant by a common covering translation.

Since $\widetilde{W}^{u}\left(\alpha_{0}\right) \cap \Theta \neq \varnothing, \alpha_{0}$ is either in the front of $\widetilde{W}^{s}(r)$ or in the back of $\widetilde{W}^{s}(u)$. If $\alpha_{0}$ is in front of $\widetilde{W}^{s}(r)$, then the argument in step 1 of Theorem 4.11 shows that the even corners in the chain of lozenges are in front of $\widetilde{W}^{s}(r)$, while the odd corners are in the back of $\widetilde{W}^{s}(u)$. If $\alpha_{0}$ is in the back of $\widetilde{W}^{s}(u)$, then the same happens with odd and even exchanged. This yields that all lozenges in the chain intersect $\widetilde{W}^{s}(r)$; hence, $q_{1}$ cannot be achieved, contradiction.

We conclude that the limit set of $g(F)$ is not a Jordan curve. As a result the same happens for $F$ and the result follows.

Lemma 5.4. Let $\Phi$ be an Anosov flow in $M^{3}$ with $\pi_{1}(M)$ negatively curved. If there is $F \in \widetilde{\mathscr{F}}^{s}$ with $\Lambda_{F}=S_{\infty}^{2}$, then $\Lambda_{L}=S_{\infty}^{2}$ for all $L \in \widetilde{\mathscr{F}}^{s}$.

Proof of 5.4. We may assume that $\mathscr{F}^{s}$ is transversely orientable. Suppose there is $L \in \widetilde{\mathscr{F}}^{s}$ with $\Lambda_{L} \neq S_{\infty}^{2}$. Let $c \in S_{\infty}^{2}-\Lambda_{L}$, and $V$ be a neighborhood of $c$ in $\widetilde{M} \cup S_{\infty}^{2}$ disjoint from $L$. By changing the orientation if necessary we may assume $V \cap \widetilde{M}$ is in front of $L$.

Let now $L^{\prime}$ be any leaf of $\widetilde{F}$. Since $\pi\left(L^{\prime}\right)$ is dense in $M$, it limits on the negative side of local sheets of $\pi(L)$. Therefore there is a covering translate $g\left(L^{\prime}\right)$ of $L^{\prime}$ which is on the negative side of $L$. Thus $g\left(L^{\prime}\right) \cap$ $V=\varnothing$ and $c \notin \Lambda_{g\left(L^{\prime}\right)}$. As a result $\Lambda_{g\left(L^{\prime}\right)} \neq S_{\infty}^{2}$, So $\Lambda_{L^{\prime}} \neq S_{\infty}^{2}$, as we wanted to prove.

Theorem 5.5. Let $\Phi$ be an Anosov flow in $M^{3}$ so that $\pi_{1}(M)$ is negatively curved. Assume that $\Phi$ is quasigeodesic. Then for any $F \in \widetilde{\mathscr{F}}^{s}$, $\Lambda_{F} \neq S_{\infty}^{2}$.

Proof of 5.5. We may assume that $\mathscr{F}^{s}, \mathscr{F}^{u}$ are transversely orientable. Then there is a double lozenge $\Theta$ in $\widetilde{M}$ (or $\mathscr{O}$ ) with unstable sides in $G, S \in \widetilde{\mathscr{F}}^{u}$, stable sides in $E, F, L \in \widetilde{\mathscr{F}}^{s}$ and pivot $p$ so that: (1) the two half leaves of $F$ are in the boundary of the lozenges of $\Theta$, (2) $G$ is in the back of $S$ and (3) $E$ is in the back of $\widetilde{W}^{u}(p)$ (see Fig. 7) (change transversal orientation to $\widetilde{\mathscr{F}}^{s}$ if necessary to produce branching of $\widetilde{\mathscr{F}}^{s}$ 


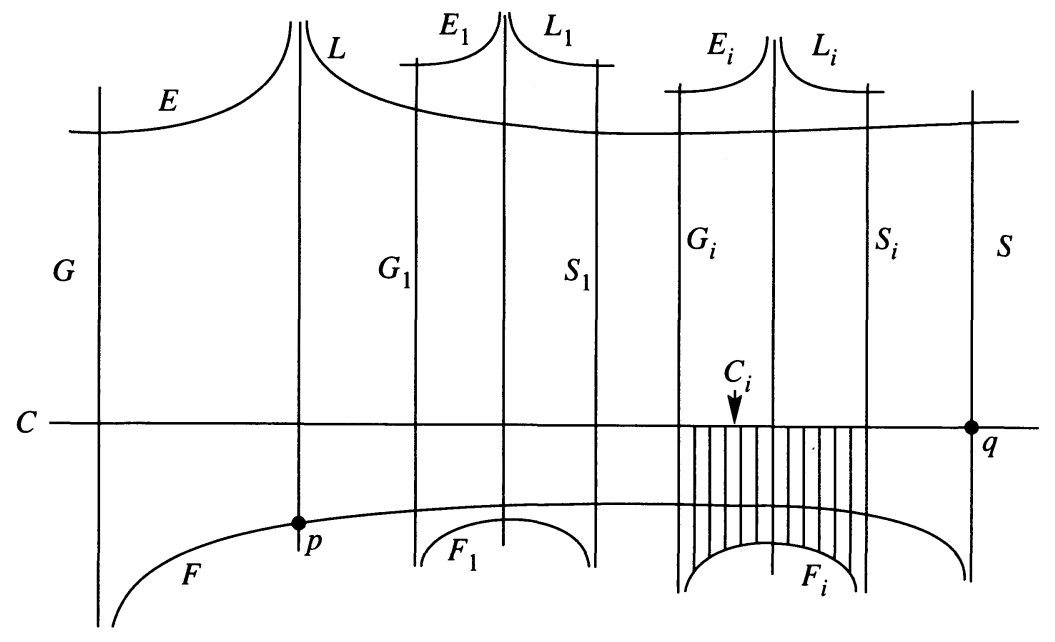

Figure 7. Sequence of lozenges.

in the positive direction). By $G$ we mean the half leaf in the boundary of $\Theta$. Thus $\pi(G)$ is dense in $M$ [13].

Choose a covering translation $g_{1}$ so that $g_{1}(G) \cap F \neq \varnothing, g_{1}(G) \cap L \neq \varnothing$. Since $g_{1}(\Theta)$ is a union of two adjacent lozenges, $g_{1}(F)$ is in the back of $F$ and $g_{1}(E)$ is in the front of $L$. Hence $g_{1}(L)$ is in the front of $L$. Finally $g_{1}(S)$ is in the front of $g_{1}(G)$, in the back of $S$ and intersects both $L$ and $F$. Inductively choose covering translations $g_{i}$ so that $g_{i}(G)$ is in the back of $S$,

$$
g_{i}(G) \cap F \neq \varnothing, \quad g_{i}(G) \cap L \neq \varnothing, \quad g_{i}(G) \rightarrow S \quad \text { as } i \rightarrow \infty,
$$

and $g_{i}(G)$ is in the front of $g_{i-1}(S)$ (see Figure 7). Notice there may be other leaves in the limit of the $g_{i}(G)$. Let $G_{i}=g_{i}(G)$ and similarly define $F_{i}, L_{i}, S_{i}$, and $E_{i}$.

Choose $C \in \widetilde{F}^{s}$ with $C \cap \Theta \neq \varnothing$. Then $C \cap S \neq \varnothing, C \cap G \neq \varnothing$ and let $C_{i}=C \cap g_{i}(\Theta)$. For any flow line $\gamma \in g_{i}(F)$, $\widetilde{W}^{u}(\gamma)$ intersects $C_{i}$ and vice versa. Hence $\eta_{-}\left(C_{i}\right)=\eta_{i}\left(g_{i}(F)\right)$. Let $q \in C \cap S$. By continuity of $\eta_{-}$there is a neighborhood $V$ of $q$ in $\widetilde{M}$ so that $\eta_{-}(V)$ is contained in a small neighborhood $U$ of $\eta_{-}(q)$ in $S_{\infty}^{2}$. As $C_{i} \cap \widetilde{W}^{s s}(q) \rightarrow q$, $\eta_{-}\left(C_{i}\right) \subset U$ for $i$ large enough. Therefore $\eta_{-}\left(g_{i}(F)\right) \subset U$ and as a result $\Lambda_{g_{i}(F)}$ is contained in the closure of $U$ and is not $S_{\infty}^{2}$. The previous lemma implies the result. 


\section{Quasigeodesic orbits}

In [13] we produced many examples of $\mathbf{R}$-covered Anosov flows in hyperbolic 3-manifolds. By Theorem 4.7 these cannot be quasigeodesic. One natural question is: are there only finitely many uniformly quasigeodesic orbits? We first notice that every closed orbit is freely homotopic to a closed geodesic and is therefore a quasigeodesic. This produces a countable number [1] of quasigeodesic orbits. Unfortunately it is hard to show they are uniformly quasigeodesic. In fact, since the union of closed orbits is dense, this would be equivalent to showing $\phi$ is quasigeodesic.

Our goal is to prove that, in general, for $K$ big enough, there are uncountably many $K$-quasigeodesic and infinitely many closed, $K$-quasigeodesic orbits of the flow. The key fact is the following localization property of quasigeodesics. Clearly any connected subset of a $K$-quasigeodesic is a local $K$-quasigeodesic. The surprising fact is that there is a converse to this.

Theorem 6.1 [21]. Fix a closed manifold $M$ with $\pi_{1}(M)$ negatively curved. Then for any $K>0$ there is $L>0$ (usually $L \gg K$ ) satisfying: if $\gamma$ is an embedded curve so that any subarc of $\gamma$ of length $\leq L$ is a $K / 2$-quasigeodesic, then $\gamma$ is $K$-quasigeodesic.

We may assume that $M$ is orientable since both properties which we want to prove are preserved under finite covers.

We now fix $\alpha$ an indivisible closed orbit of $\Phi$, and let $\tau_{1}$ be a flow segment of $\Phi$ starting and ending very near the same point $p$ of $\alpha$. We assume that $\tau_{1}$ is not contained in a small neighborhood of $\alpha$. Let $\tau_{0}$ be a fixed small segment from $p$ to $\tau_{1}(0)$ and $\tau_{2}$ another from $\tau_{1}(1)$ to $p$. Then $\tau=\tau_{0} \tau_{1} \tau_{2}$ is a closed loop based at $p$. For simplicity of notation we will omit the $*$ 's in $\tau_{0} * \tau_{1} * \tau_{2}$, etc.

Let $\mathscr{A}$ be the set of all sequences $\xi=\left(m_{i}\right)_{i \in A}$ such that the following hold:

(1) $A$ is an interval in the integers that contains $\{0,1\}$, i.e., $A=\{j \in$ $\left.\mathbf{Z} \mid n_{0}<j<n_{1}\right\}$, where $-\infty \leq n_{0}<0$ and $1<n_{1} \leq \infty$.

(2) Each term $m_{i}$ is in $\mathbf{N}^{*} \cup\{\infty\} \quad\left(\mathbf{N}^{*}=\mathbf{N}-\{0\}\right)$.

(3) Only the first and the last terms of the sequence may be $\infty$. Hence $m_{i}=\infty$ does not occur in bi-infinite sequences.

For $r \in \mathbf{N}$, let

$$
\mathscr{A}_{r}=\left\{\xi \in \mathscr{A} \mid m_{i} \geq r, \forall i \in A\right\} .
$$

Finally, given $\xi \in \mathscr{A}$, let $\gamma_{\xi}$ be the (possibly infinite) path defined by

$$
\gamma_{\xi}=\cdots \alpha^{m_{-1}} \tau \alpha^{m_{0}} \tau \alpha^{m_{1}} \cdots
$$


where $\alpha^{\infty}$ in $\tau \alpha^{\infty}$ is just the infinite path starting at the endpoint of $\tau$ and going along $\alpha$ infinitely many times in the forward direction, while in $\alpha^{\infty} \tau$ it is the infinite path ending in the initial point of $\tau$ and going backwards along $\alpha$ infinitely many times. The path $\gamma_{\xi}$ is constructed starting with the initial point of $\tau$ (between $\alpha^{m_{0}}$ and $\alpha^{m_{1}}$ ) and inductively constructing both directions. Let $p \alpha^{\infty}$ be the path starting at $p$ and going forward along $\alpha$ infinitely many times and similarly define $\alpha^{\infty} p$.

If the endpoints of $\tau$ are sufficiently near $\alpha$ then, by the shadowing lemma [1], [6], [7], any $\gamma_{\xi}$ is shadowed by a true orbit $\beta_{\xi}$, which may be finite or not. Expansiveness [6], [32] implies that if $\gamma_{\xi}$ is infinite in both directions, then $\beta_{\xi}$ is unique. Furthermore $\beta_{\xi} \neq \alpha$ for any $\xi$ because $\tau_{1}$ is not very near $\alpha$. Our goal is to show that the orbits $\beta_{\xi}$ are quasigeodesics. Notice that $\gamma_{\xi}$ and $\beta_{\xi}$ are always uniformly very close to each other throughout their lengths, so in order to prove the uniform quasigeodesic behavior of $\beta_{\xi}$ it suffices to do this for $\gamma_{\xi}$.

Lemma 6.2. If $\xi_{0}=\left(m_{0}, m_{1}\right)=(\infty, \infty)$, then $\gamma_{\xi_{0}}$ is a $K$-quasigeodesic for some $K$ :

Proof of 6.2. Fix a base point $\tilde{p} \in \widetilde{M}$ with $\pi(\tilde{p})=p$. Let $\tilde{\alpha}_{0}$ be the lift of $\alpha^{\infty} p$ ending at $\tilde{p}$. Let $\tilde{\tau}$ be the lift of $\tau$ starting in $\tilde{p}$, ending in $\tilde{q}$ and let $\tilde{\alpha}_{1}$ be the lift of $p \alpha^{\infty}$ starting in $\tilde{q}$. Then $\tilde{\gamma}=\tilde{\alpha}_{0} \tilde{\tau} \tilde{\alpha}_{1}$ is a lift of $\gamma_{\xi_{0}}$. Since $\alpha$ is a quasigeodesic in $M, \tilde{\gamma}$ has well-defined limit points.

We first show that the positive and negative limit points of $\tilde{\gamma}$ are distinct. Let $g, h$ be the covering translations of $\widetilde{M}$ (with basepoint $\tilde{p}$ ) associated to $\alpha$ and $\tau$. Let $a \neq b$ be the fixed points of $g$ in $S_{\infty}^{2}$, where $a$ is the attracting fixed point. The positive limit point of $\tilde{\gamma}$ is $h(a)$, and its negative limit point is $b$. If $h(a)=b$, then $h^{-1} g h(a)=a=g(a)$. But as the stabilizer of a point in $S_{\infty}^{2}$ is cyclic [21], there is an indivisible covering translation $f$ with $h^{-1} g h=f^{j_{0}}$ and $g=f^{j_{1}}$. Since $M$ is orientable, it follows that $f$ fixes both $a$ and $b$. Furthermore $f^{j_{1}}(h(b))=h(b)$, hence either $h(b)=a$ or $h(b)=b$. But $h(a)=b$ so we must have $h(b)=a$. Then $h^{2}(a)=a$, so as above this implies that $h$ fixes $a$, contradiction. Therefore the endpoints of $\tilde{\gamma}$ are distinct.

Let $\tau^{\prime} \subset \widetilde{M}$ be a minimal geodesic connecting the endpoints of $\tilde{\gamma}$. Project $\tilde{\gamma}$ to $\tau^{\prime}$ as follows: $\tilde{\alpha}_{0}$ is $k_{0}$-quasigeodesic for some $k_{0}$, therefore project $\tilde{p}$ to the nearest point in $\tau^{\prime}$ and define $\theta: \tilde{\alpha}_{0} \rightarrow \tau^{\prime}$ continuous so that $d(\theta(u), u)<R$ (for some $R$ ) and

$$
d_{0}(u, v)<b_{0}+b_{1} d(\theta(u), \theta(v)),
$$

where $d_{0}$ is measured along $\tilde{\gamma}$. This can be done in two steps: first, using 


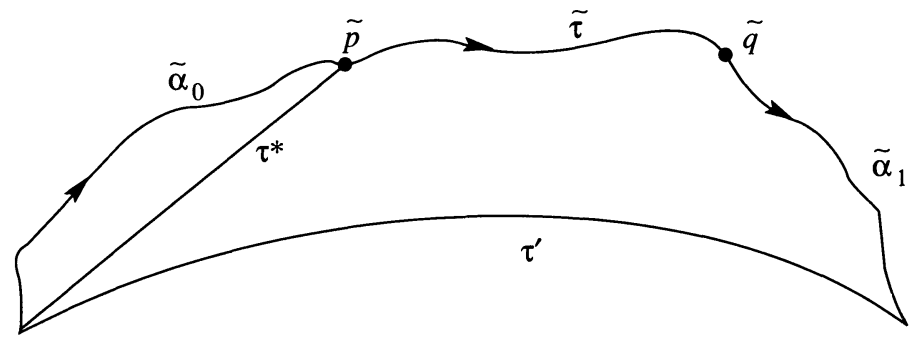

Figure 8. A basic quasi geodesic.

quasigeodesic behavior of $\tilde{\alpha}_{0}$, project $\tilde{\alpha}_{0}$ to a geodesic ray $\tau^{*}$ ending in $\tilde{p}$ and with same limit point as $\tilde{\alpha}_{0}$, in a way satisfying the above inequality. Then project $\tau^{*}$ to $\tau^{\prime}$ so as to multiply all lengths by a constant. Do the same for $\tilde{\alpha}_{1}$, projecting $\tilde{q}$ to $\theta(\tilde{p})$. Then project $\tilde{\tau}$ to $\theta(\tilde{p})$. See Figure 8.

Let $b_{2}$ be the length of $\tilde{\tau}$. Let $u, v \in \tilde{\gamma}$, with $u \in \tilde{\alpha}_{0}$ and $v \in \tilde{\alpha}_{1}$. Then

$$
\begin{aligned}
d_{0}(u, v) & =d_{0}(u, \tilde{p})+d_{0}(\tilde{p}, \tilde{q})+d_{0}(\tilde{q}, v) \\
& \leq b_{0}+b_{1} d(\theta(u), \theta(\tilde{p}))+b_{2}+b_{0}+b_{1} d(\theta(\tilde{q}), \theta(v)) \\
& =\left(2 b_{0}+b_{2}\right)+b_{1} d(\theta(u), \theta(v)) \\
& <\left(2 b_{0}+b_{2}+2 b_{1} R\right)+b_{1} d(u, v) .
\end{aligned}
$$

Since $d(u, v)$ is clearly $\leq d_{0}(u, v)$, there is a uniform quasi-isometric relation between $d(u, v)$ and $d_{0}(u, v)$. The other cases, for instance if both $u, v$ are in $\tilde{\alpha}_{0}$, are simpler. Therefore there is a global quasiisometric relation, and $\gamma_{\xi_{0}}$ is quasigeodesic.

Proposition 6.3. For $K$ big enough, there is $r_{0}$ so that all orbits $\beta_{\xi}$ with $\xi$ in $\mathscr{A}_{r_{0}}$ are $K$-quasigeodesics.

Proof of 6.3. By the previous lemma, $\gamma_{\xi_{0}}$ is a $K / 2$-quasigeodesic for some $K$. Using Theorem 6.1 we find $L$ so that any $(K / 2, L)$ local quasigeodesic is a global $K$-quasigeodesic. Choose $r_{0}$ so that $l\left(\alpha^{r_{0}}\right)>L$. Let $\xi \in \mathscr{A}_{r_{0}}$ and $\alpha^{\prime}$ a segment in $\gamma_{\xi}$ of length $\leq L$. Since $l\left(\alpha^{r_{0}}\right)>L$, it follows that for some $i \in A$, one of the following must happen: $\alpha^{\prime} \subset \alpha^{m_{i}}$, $\alpha^{\prime} \subset \alpha^{m_{i}} \tau, \alpha^{\prime} \subset \tau \alpha^{m_{i}}$, or $\alpha^{\prime} \subset \alpha^{m_{i}} \tau \alpha^{m_{i+1}}$. In any case, for some $i \in A$,

$$
\alpha^{\prime} \subset \alpha^{m_{i}} \tau \alpha^{m_{i+1}} \subset \alpha^{\infty} \tau \alpha^{\infty}=\gamma_{\xi_{0}},
$$

which is a $K / 2$-quasigeodesic. Hence $\gamma_{\varepsilon}$ is a $(K / 2, L)$ local quasigeodesic. This implies that $\gamma_{\xi}$, hence $\beta_{\xi}$, is a $K$-quasigeodesic.

Theorem 6.4. Let $\Phi$ be an Anosov flow in $M^{3}$ so that $\pi_{1}(M)$ is negatively curved. Then for big enough $K$, there are uncountably many orbits 
of $\Phi$, which are $K$-quasigeodesics and infinitely many closed orbits in this set.

Proof of 6.4. Let $K, r_{0}$ given by the previous proposition. Let $\widehat{\mathscr{A}_{r_{0}}}$ be those $\xi$ in $\mathscr{A}_{r_{0}}$ so that $\xi=\left(m_{i}\right)_{i \in A}$ satisfies

$$
\sum_{i \leq 0} m_{i}=\infty \text { and } \sum_{i \geq 1} m_{i}=\infty
$$

For such $\xi$, there is a unique $\beta_{\xi}$ which will be a full orbit. By the proposition, $\beta_{\xi}$ is a $K$-quasigeodesic. The proof now follows from symbolic dynamics. For any periodic sequence $\xi \in \widehat{\mathscr{A}}_{r_{0}}, \beta_{\xi}$ is a periodic orbit. If two sequences in $\widehat{\mathscr{A}}_{r_{0}}$ are not shift equivalent, the closed orbits are different. This shows there are infinitely many $K$-quasigeodesic closed orbits. Furthermore since there are uncountably many shift inequivalent sequences in $\widehat{\mathscr{A}}_{r_{0}}$, it follows that there are uncountably many distinct $K$-quasigeodesic orbits.

Remark. Notice that in this construction, all orbits are in a small neighborhood of $\tau \cup \alpha$ !

\section{Acknowledgements}

We thank Bill Thurston for his encouragement and many ideas concerning this paper, in particular for suggesting the construction in $\S 6$. Thanks also to Joe Christy for his help with intransitive Anosov flows, to Lee Mosher for ideas concerning pseudo-Anosov flows and to Francis Bonahon for help with negatively curved groups. Finally we thank the referee for numerous suggestions, which greatly helped clarify the exposition of the paper.

\section{References}

[1] D. V. Anosov, Geodesic flows on closed Riemannian manifolds with negative curvature, Proc. Steklov Inst. Math. 90 (1969).

[2] D. V. Anosov \& Y. Sinai, Some smooth ergodic systems, Russian Math. Surveys 22 (1967) 103-167.

[3] M. Bestvina \& J. Mess, The boundary of negatively curved groups, J. Amer. Math. Soc. 4 (1991) 469-481.

[4] C. Bonatti \& R. Langevin, Un exemple de flot d'Anosov transitif transverse à un tore et non conjugué à une suspension, Prepublication Université de Bourgogne, 1992.

[5] R. Bowen, Periodic orbits for hyperbolic flows, Amer. J. Math. 94 (1972) 1-30.

[6] __ Symbolic dynamics for hyperbolic flows, Amer. J. Math. 95 (1973) 429-460.

[7] _ Equilibrium states and the ergodic theory of Anosov diffeomorphisms, Lecture Notes in Math., 470, Springer, Berlin, 1975. 
[8] J. Cannon \& W. Thurston, Group invariant peano curves (to appear).

[9] J. Christy, Intransitive Anosov flows on 3-manifolds (to appear).

[10] C. Conley, Isolated invariant sets and the Morse index, Regional Conf. Ser. in Math., 38, Amer. Math. Soc., Providence, RI, 1978.

[11] S. Fenley, Quasi-isometric foliations, Topology 31 (1992) 667-676.

[12] __, Asymptotic properties of depth-one foliations in hyperbolic 3-manifolds, J. Differential Geometry 36 (1992) 269-313.

[13] __, Anosov flows in 3-manifolds, Ann. of Math. (2) 139 (1994) 79-115.

[14] _ Continuous extension of Anosov foliations, preprint.

[15] J. Franks \& R. Williams, Anomalous Anosov flows, Global Theory of Dynamical Systems, Lecture Notes in Math., 819, Springer, Berlin, 1980.

[16] D. Fried, Transitive Anosov flows and pseudo-Anosov maps, Topology 22 (1983) 299303.

[17] D. Gabai, Foliations and the topology of 3-manifolds, J. Differential Geometry 18 (1983) 445-503.

[18] D. Gabai \& U. Oertel, Essential laminations and 3-manifolds, Ann. of Math. (2) 130 (1989) 41-73.

[19] E. Ghys \& P. de la Harpe (Editors), Sur les groupes hyperboliques d'aprés Mikhael Gromov, Progress in Math., 83, Birkhäuser, Basel, 1991.

[20] S. Goodman, Dehn surgery and Anosov flows, Proc. Geometric Dynamics Conf., Lecture Notes in Math., 1007, Springer, Berlin, 1983.

[21] M. Gromov, Hyperbolic groups, Essays on Group Theory, Springer, 1987, 75-263.

[22] J. Hempel, 3-manifolds, Annals of Math. Studies, 86, Princeton University Press, Princeton, NJ, 1976.

[23] H. Imanishi, On the theorem of Denjoy-Sacksteder for codimension one foliations without holonomy, J. Math. Kyoto Univ. 14 (1974) 607-634.

[24] W. Jaco, Lectures on 3-manifold topology, CBMS Regional Conf. Ser. in Math., 43, Amer. Math. Soc., Providence, RI, 1980.

[25] J. Morgan, On Thurston's uniformization theorem for 3 dimensional manifolds. The Smith conjecture (J. Morgan and H. Bass, eds.), Academic Press, San Diego, 1984, $37-125$.

[26] L. Mosher, Dynamical systems and the homology norm of a 3-manifold. I: Efficient intersection of surfaces and flows, Duke Math. J. 65 (1992) 449-500.

[27] _ Dynamical systems and the homology norm of a 3-manifold. II, Invent. Math. 107 (1992) 243-281.

[28] __ Examples of quasigeodesic flows on hyperbolic 3-manifolds, Proc. Ohio State Univ. Research Semester on Low-Dimensional Topology, de Gruyter, Berlin, 1992.

[29] S. P. Novikov, Topology of foliations, Trans. Moscow Math. Soc. 14 (1963) 268-305.

[30] F. Palmeira, Open manifolds foliated by planes, Ann. of Math. (2) 107 (1978) 109-131.

[31] J. Plante, Anosov flows, Amer. J. Math. 94 (1972) 729-754.

[32] M. Shub, Global stability of dynamical systems, Springer, Berlin, 1987.

[33] S. Smale, Differentiable dynamical systems, Bull. Amer. Math. Soc. 73 (1967) 747-817.

[34] D. Sullivan, Cycles for the dynamical study of foliated manifolds and complex manifolds, Invent. Math. 36 (1976) 225-255.

[35] W. Thurston, The geometry and topology of 3-manifolds, Princeton Univ. Lecture Notes, 1982.

Mathematical Sciences Research Institute and UNIVERSITY OF CALIFORNIA, BERKELEY 